Article

\title{
A New Cycle-Slip Repair Method for Dual-Frequency BDS Against the Disturbances of Severe Ionospheric Variations and Pseudoranges with Large Errors
}

\author{
Dehai Li ${ }^{1}$, Yaming Dang ${ }^{1}$, Yunbin Yuan ${ }^{2}$ and Jinzhong Mi ${ }^{1, *}$ \\ 1 Chinese Academy of Surveying and Mapping, No.28, Lianhuachi West Road, Beijing 100830, China; \\ Lidh@casm.ac.cn (D.L.); Dangym@casm.ac.cn (Y.D.) \\ 2 Innovation Academy for Precision Measurement Science and Technology, Chinese Academy of Science, \\ Xiaohongshan West Road, Wuhan 430071, China; yybgps@asch.whigg.ac.cn \\ * Correspondence: goldheal@casm.ac.cn; Tel.: +86-186-0123-3503
}

check for

updates

Citation: Li, D.; Dang, Y.; Yuan, Y.; Mi, J. A New Cycle-Slip Repair Method for Dual-Frequency BDS Against the Disturbances of Severe Ionospheric Variations and Pseudoranges with Large Errors. Remote Sens. 2021, 13, 1037. https:// doi.org/10.3390/rs13051037

Academic Editor:

Amir Khodabandeh

Received: 4 February 2021

Accepted: 8 March 2021

Published: 9 March 2021

Publisher's Note: MDPI stays neutral with regard to jurisdictional claims in published maps and institutional affiliations.

Copyright: (C) 2021 by the authors Licensee MDPI, Basel, Switzerland. This article is an open access article distributed under the terms and conditions of the Creative Commons Attribution (CC BY) license (https:// creativecommons.org/licenses/by/ $4.0 /)$.

\begin{abstract}
Many Beidou navigation satellite system (BDS) receivers or boards provide dual-frequency measurements to conduct precise positioning and navigation for low-power consumption. Cycleslip processing is a primary work to guarantee consistent, precise positioning with the phase data. However, the cycle-slip processing of BDS dual-frequency phases still follows with those of existing GPS methods. For single-satellite data, cycle-slip detection (CSD) with the geometry-free phase (GF) is disturbed by severe ionospheric delay variations, while CSD or cycle-slip repair (CSR) with the Melbourne-Wubbena combination (MW) must face the risk of the tremendous disturbance from large pseudorange errors. To overcome the above limitations, a new cycle-slip repair method for BDS dual-frequency phases (BDCSR) is proposed: (1) An optimal model to minimize the variance of the cycle-slip calculation was established to the dual-frequency BDS, after correcting the ionospheric variation with a reasonable and feasible way. (2) Under the BDS dual-frequency condition, a discrimination function was built to exclude the adverse disturbance from the pseudorange errors on the CSR, according to the rankings of the absolute epoch-difference GFs calculated by the searched cycle-slip candidates after correcting the ionospheric variation. Subsequently, many compared CSR tests were implemented in conditions of low and medium elevations during strong geomagnetic storms. Comparisons from the results of different methods show that: (1) The variations of ionospheric delays are intolerable in the cycle-slip calculation during the geomagnetic storm, and the tremendous influence from the ionospheric variation should be corrected before calculating the cycle-slip combination with the BDS dual-frequency data. (2) Under the condition of real dual-frequency BDS data during the geomagnetic storm, the actual success rate of the conventional dual-frequency CSR (CDCSR) by employing the optimized combinations, but absenting from the discrimination function, is lower than that of BDCSR by about $\%$; The actual success rate of the CSD with MW (MWCSD), is lower than that of BDCSR by about $\%$. (3) After adding gross errors of $0.7 \mathrm{~m}$ to all real epoch-difference pseudoranges epoch-by-epoch, results of CDCSR and MWCSD showed many errors. However, BDCSR achieved a higher actual success rate than those of CDCSR and MWCSD, about 43\% and 16\%, respectively, and better performance of refraining the disturbance of large pseudorange error on the cycle-slip determination was achieved in the BDCSR methodology.
\end{abstract}

Keywords: dual-frequency BDS; cycle-slip repair; cycle-slip detection; cycle-slip discrimination; ionospheric variation

\section{Introduction}

Global navigation satellite system (GNSS) relies on using phase observations to provide precise services of positioning, timing, navigation, orbit determination, clock error estimation, and so on [1,2]. The continuous phase observations without abnormal slips help to guarantee the consistency and convergence of the aforementioned precise services. 
Unfortunately, when the phase observation was interrupted or relocked, cycle slips will appear in the phase data at the subsequent epoch. On one hand, if the cycle slip is not correctly detected and reasonably handled, the results of GNSS precise services will be destroyed [3,4]. On the other hand, if the cycle slip is not repaired, there may cause the reinitialization of the ambiguity parameter $[5,6]$. When more satellites experienced cycle slips at the same epoch, the initialization procedure would give rise to the extra costs of waiting for convergence and degrading of service accuracy [7]. Therefore, cycle-slip detection (CSD) or cycle-slip repair (CSR) has become the basic task of GNSS data processing.

As one of the main GNSS, the third-generation BDS has completed global networking and is providing triple-frequency navigation signals. The B1 and B2 frequencies are mainly assigned for BDS's civil signals and services. In order to pursue low-power consumption, some popular GNSS boards or receivers, such as Trimble BD970, Trimble MB2, Novatel OEM7600, Novatel OEM7720, and Novatel OEM6, still choose to observe and output BDS dual-frequency phases and pseudoranges. In addition, many commissioned receivers, such as Trimble R9 and Leica GR25 with low-version firmware, merely provide BDS dual-frequency data. Therefore, it is necessary to pay attention to the works of BDS dual-frequency CSD and CSR.

Currently, BDS cycle-slip processing mainly follows the GPS dual-frequency CSD and CSR methods. For multi-satellite dual-frequency data, Lacy et al. (2012) and Li et al. (2019c) established a rigorous uncombined epoch-difference observation model, and the cycleslip value was obtained through parameter estimation [8,9]. Li et al. (2019a) improved the reliability of the cycle-slip estimation via a mathematic prediction of ionospheric variations [3]. Li et al. (2019b) filtered multi-epoch data so as to get more precise cycle-slip values [4]. Moreover, those methods can be extended and adapted to the cases of different systems and frequencies.

For single-satellite data, CSD mainly resorts to the methods of outlier detection. Lacy et al. (2008) exploited the Bayesian approach to estimate, and correct cycle slips with geometry-free phase (GF) [10]. Mertikas and Rizos (1997) provided a statistical accumulation test to detect phase mutation [11]. Blewitt (1990) used dual-frequency GF to detect cycle slip [12]. However, GF still contains the effects of ionospheric delay. Large ionospheric variations will lead to false CSD results, so the premise is demanded the quiet or slowly changing ionosphere $[13,14]$. Generally, the Melbourne-Wubbena combination (MW) is applied to calculate the cycle-slip combination and serve the CSD and CSR $[15,16]$. Nevertheless, the pseudoranges are included in the calculation of the MW. Large pseudorange errors will cause fatal biases in the MW and result in CSD and CSR failures.

Most mature GNSS software utilizes the CSD with GF (GFCSD) and ambiguity reinitialization to deal with cycle slips. Liu (2011) used the hybrid of GF and MW to estimate and determine cycle slip [17]. In order to reduce the influence of large pseudorange errors, Cai et al. (2013) designed a method of filtering multi-epoch data to improve the precision of the calculated cycle-slip combination [18]. However, the filtering method requires that all observation data in the filter window should not contain any cycle slips. This premise is hard to be fully satisfied in real-time practice because it is difficult to ensure that the data from current and subsequent epochs have no cycle slips in real-time CSR.

In response to the above limitations, this study proposed a cycle-slip repair method for BDS dual-frequency phases (BDCSR) to overcome the adverse disturbances of severe ionospheric variations and large pseudorange errors on CSR. Consequently, the following contributions would be achieved by BDCSR. Compared to GFCSD, BDCSR proposed a more reasonable and feasible method to correct the ionospheric variations. Via correcting the ionospheric variations, a model for minimizing the variance of the cycle-slip combination was established to the dual-frequency BDS. Relative to MW, a more precise method was provided to calculate the cycle-slip combination. Supplementary to the conventional dual-frequency CSR with the optimized combinations (CDCSR), a discrimination function is constructed to complete the selection and determination of the final cycle slips. This 
function not only eliminates the influence of pseudorange error but also presents a sensitive response to wrong cycle slips and a notable discriminating ability.

BDCSR will be tested at low-latitude and high-latitude regions during the weather of a geomagnetic storm. The testing conditions include the medium elevations and low elevations, the actual low-dynamic geosynchronous earth orbit (GEO) satellite, the highdynamic medium earth orbit (MEO) satellite and the inclined geosynchronous orbit (IGSO) satellite, the real normal data, and the real data of adding gross errors on the pseudoranges. The methods and results were compared among the BDCSR, CDCSR, and the cycle-slip processing methods with MW. Both BDCSR and CDCSR used the optimized combinations. The difference is that the BDCSR methodology constructed a discrimination function to search and determine the correct cycle-slip values, but CDCSR output the directly rounding integers from the calculated cycle-slip combinations without the help of the discrimination function. The following parts are arranged as: First, the BDCSR method was established; Then, via designing the comparative CSR tests, the testing results were analyzed; Finally, the characteristics and improvement of the BDCSR methodology were evaluated and concluded.

\section{Methods}

With the following steps, we built the BDCSR methodology. First, by analyzing the disturbance of ionospheric variation on the CSD with GF, a practical strategy was investigated to correct the ionospheric variation. Second, as a result of minimizing the variance of the cycle-slip combination, a high-precision way was supplied for calculating the cycle-slip combinations after correcting the ionospheric variation. Finally, for the sake of refraining from the disturbance of pseudorange error on CSR, a discrimination function under the condition of dual-frequency BDS was proposed with the epoch-difference GF after correcting the ionospheric variation.

\subsection{Conventional Cycle Slip Calculation with GF}

The phases and pseudoranges from BDS dual-frequency receivers are denoted as follows: $\phi_{j}$ is the phase; $j=1,2$ are the indexes on B1 and B2 frequencies, respectively; $p_{j}$ is the pseudorange; $\lambda_{j}$ is the wavelength, and $f_{j}$ is the frequency value. The GF consisting of BDS dual-frequency phases can be formed as:

$$
L_{4}=\phi_{1} \lambda_{1}-\phi_{2} \lambda_{2}
$$

where GF displays the characteristic of offsetting the geometric elements independent on different frequencies, but the ionospheric delay is reserved in the GF with the ionospheric scaling factor (ISF) $0.672=f_{1}^{2} / f_{2}^{2}-1$ relative to B1 with a unit of $\mathrm{m}$. Therefore, the GFCSD is only suitable for a quiet ionospheric environment; that is, the ionospheric variation between consecutive epochs should be close to 0 . However, the ionosphere will change tremendously in the geomagnetic storm or other active ionospheres. Hence, the correction of ionospheric variation will be introduced in the next section.

\subsection{Correction of Ionospheric Variation and Its Precision}

The ionospheric delay on B1 can be calculated with the BDS dual-frequency phases by [19]:

$$
\text { iono }_{1}=\frac{\left(\phi_{1}+\mathrm{N}_{1}\right) \lambda_{1}-\left(\phi_{2}+\mathrm{N}_{2}\right) \lambda_{2}}{1-\mathrm{f}_{1}^{2} / \mathrm{f}_{2}^{2}}
$$

where $N_{j}$ is the ambiguity. By the epoch-difference treatment, the variation of ionospheric delay between adjacent epochs is obtained as:

$$
\Delta \text { iono }_{1, \mathrm{k}}=\text { iono }_{1, \mathrm{k}}-\text { iono }_{1, \mathrm{k}-1}
$$


where $\Delta$ is the epoch-difference operator, and iono ${ }_{1, k}$ is the ionospheric delay at $\mathrm{k}$ epoch on the frequency $f_{1}$. If there is no cycle slip, the ionospheric variation can be gained by:

$$
\Delta \text { iono }_{1, \mathrm{k}}=\frac{\Delta \phi_{1} \lambda_{1}-\Delta \phi_{2} \lambda_{2}}{1-\mathrm{f}_{1}^{2} / \mathrm{f}_{2}^{2}}
$$

If the observations between different frequencies and epochs are independent, the variance of the ionospheric variation is simplified as:

$$
\delta_{1, \Delta \text { iono }}^{2}=\frac{\delta_{\Delta \phi_{1}}^{2} \lambda_{1}^{2}+\delta_{\Delta \phi_{2}}^{2} \lambda_{2}^{2}}{\left(1-\mathrm{f}_{1}^{2} / \mathrm{f}_{2}^{2}\right)^{2}}
$$

where $\delta_{\Delta \phi_{j}}=\sqrt{2} \delta_{\phi_{j}}$ is the standard deviation (STD) of $\Delta \phi_{j}$, and $\delta_{\phi_{j}}$ is the STD of $\phi_{j}$ with 0.01 cycles.

Obviously, the ionospheric variation, embodied as the first-order difference of ionospheric delays between adjacent epochs, can be calculated by the epoch-difference phases. Using the real BDS dual-frequency data at a low-latitude region with an active ionosphere, the first-order differences of ionospheric delays $\Delta \mathrm{iono}_{1, \mathrm{k}}$ from MEO Satellite 11 are calculated with (4) and shown in the upper parts of Figure 1. For the real data with an interval of $30 \mathrm{~s}$ under the environment where a 7-level geomagnetic storm occurred, the epochdifference ionospheric delays exhibit that mean of ionospheric variations is about $0.029 \mathrm{~m}$, and the maximum value can reach $0.08 \mathrm{~m}$. It indicates that the ionospheric delay presents a significant influence even though the epoch-difference operation has been enforced. In the GF with the reduced ISF as low as 0.672 times, GFCSD treats the influence of the ionospheric variation to be negligible. However, in the combinations of BDS dual-frequency phases and pseudoranges, the effect of ionospheric delay will be amplified more than 10 times. If the ionospheric variation is not corrected, it will bring a tremendous disturbance to the combinations of phases and pseudoranges.

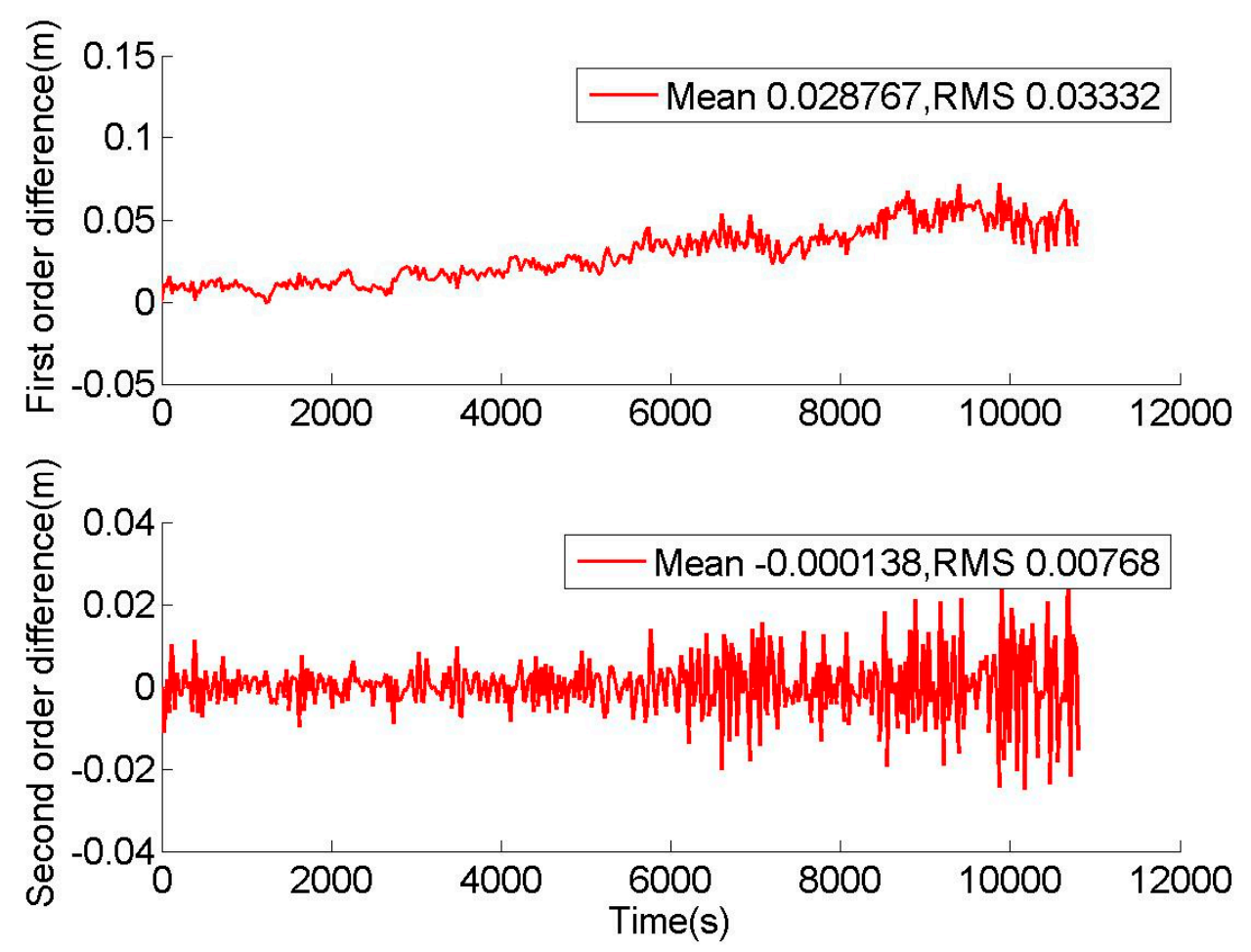

Figure 1. The first-order and second-order differences of the ionospheric delays on the high-dynamic medium earth orbit (MEO) Satellite 11 during the geomagnetic storm, which was calculated by the Beidou navigation satellite system (BDS) dual-frequency data, started from UTC 8 May 2016 00:00:00 with an interval of $30 \mathrm{~s}$ at the CUT0 station. 
The characteristics of ionospheric variations can be carefully investigated from the view of the second-order differences of ionospheric delays $\Delta \Delta \mathrm{iono}_{1, \mathrm{k}}=\Delta \mathrm{iono}_{1, \mathrm{k}}-\Delta \mathrm{iono}_{1, \mathrm{k}-1}$. Similarly, the second-order differences of ionospheric delays are calculated and shown in the bottom parts of Figure 1. The mean of the second-order differences of ionospheric delays is about 0.00014 , and the ratio between the mean of the first-order differences and that of the second-order differences is about 207. It demonstrates that $\Delta \Delta$ iono $_{1, \mathrm{k}} \rightarrow 0$ and the ionospheric variation at the previous epoch can be adopted to correcting the current ionospheric variation.

As the applicable restriction, GFCSD assumes that the first-order differences of ionospheric delays approach 0 . This paper relaxes this restriction. BDCSR supposes the second-order differences of ionospheric delays are close to 0 so that a more reasonable and practical method is obtained to correct the ionospheric variation. In this manner, the ionospheric variation at the current epoch is corrected by that of at the previous epoch calculated by the observed dual-frequency phases. As the initialized condition, the first-epoch phase data without cycle slip are required to be sought out. In this situation, the GFCSD can be used to find the initial epoch of phase data without cycle slip.

BDCSR provides a simple and effective method to correct the ionospheric variation. By employing the ionospheric variation calculated on the previous epoch to correct the ionospheric variation on the current epoch, and the correction residual is embodied in the second-order ionospheric delay. The tolerable limit is 0.015 in GFCSD, which is three times the STD of the epoch-difference GF. After correcting the ionospheric variation in the epoch-difference GF, when the influence of the residual from the ionospheric variation correction on the epoch-difference GF is greater than 0.015, the GFCSD after correcting the ionospheric variation deems that the cycle slip has occurred. According to the limited value of 0.015 and the ionospheric scaling factor of 0.672 , the tolerable error of ionospheric variation correction is limited to be $0.022 \mathrm{~m}$ on B1. That is, when the second-order total electron content (TEC) on B1 is less than 0.133 TEC unit (TECU), the corrected GFCSD is still applicable to the phase data under this ionospheric variation environment. Summarily, this method weakens the influence of ionospheric variation on the cycle-slip calculation, especially in the case of calculating cycle-slip combination with the BDS dual-frequency phases and pseudoranges. Then, the residuals after the ionospheric correction will be handled in the subsequent section.

\subsection{Optimal Cycle Slip Calculation with BDS Dual-Frequency Data}

The multipath effect, phase bias, and code bias are almost constant between adjacent epochs and would be eliminated via epoch-difference operation [20-22]. The ionospheric variation has been corrected. Thus, the cycle-slip combination can be calculated with the epoch-difference combination of BDS dual-frequency phases and pseudoranges by:

$$
\Delta N_{c}=\frac{\Delta p_{c}}{\lambda_{c}}-\Delta \phi_{c}-\text { ISF } \Delta \text { iono }_{1}
$$

where $\Delta$ is the epoch-difference operator; $\phi_{c}=m_{1} \phi_{1}+m_{2} \phi_{2}$ is the phase combination; $m_{\mathrm{j}}$ is the coefficients of phase combination; $\mathrm{c}$ is the velocity of light; and $\lambda_{c}=c /\left(m_{1} f_{1}+m_{2} f_{2}\right)$ is the combined wavelength. $p_{c}=n_{1} p_{1}+n_{2} p_{2}$ is the pseudorange combination and $n_{j}$ is the coefficients of the pseudorange combination. With respect to B1 on the unit of $\mathrm{m}$, $I S F P=n_{1}+n_{2} f_{1}^{2} / f_{2}^{2}$ is the ISF in the pseudorange combination; with respect to B1 on the unit of the cycle, ISFC $=m_{1} / \lambda_{1}+m_{2} f_{1}^{2} /\left(\lambda_{2} f_{2}^{2}\right)$ is the ISF in the phase combination; and $I S F=I S F P / \lambda_{c}+I S F C$ is the ISF in the cycle-slip combination with the unit of the cycle. The ionospheric variation on $\mathrm{B} 1$ denoted as $\Delta \mathrm{iono}_{1}$ would be corrected by (4) calculated with the epoch-difference phase data at the previous epoch. 
If the observations are independent on different frequencies and epochs, the variance of the cycle-slip combination is simplified as:

$$
\sigma_{\Delta N_{c}}^{2}=\frac{\sigma_{\Delta p_{c}}^{2}}{\lambda_{c}^{2}}+\sigma_{\Delta \phi_{c}}^{2}+\sigma_{1, \Delta \text { iono }}^{2} I S F^{2}
$$

where $\sigma_{\Delta \phi_{c}}=\sqrt{\left(m_{1}^{2} \sigma_{\Delta \phi_{1}}^{2}+m_{2}^{2} \sigma_{\Delta \phi_{2}}^{2}\right)}$ is the STD of $\Delta \phi_{c} ; \sigma_{\Delta P_{c}}=\sqrt{n_{1}^{2} \sigma_{\Delta p_{1}}^{2}+n_{2}^{2} \sigma_{\Delta p_{2}}^{2}}$ is the STD of $\Delta p_{c} ; \delta_{\Delta p_{j}}=\sqrt{2} \delta_{p_{j}}$ is the STD of $\Delta p_{j}$, and $\delta_{p_{j}}$ is the STD of $p_{j}$ with $0.3 \mathrm{~m}$; and $\sigma_{1, \Delta \text { iono }}$ is the STD of the ionospheric variation correction on the frequency $f_{1}$, shown in (5) with the unit of $\mathrm{m}$.

The factors involved in the variance of the cycle-slip calculation can be analyzed from (7). It demonstrates that the longer wavelength, the lower levels of combination noises, and the smaller ISF, as well as ionospheric residual after the correction, could benefit from the higher precision of the cycle-slip calculation. Taking into account the aforementioned beneficial requirements, an optimization model is formed to minimize the variance of the cycle-slip combination by (8), along with the geometry-free constraint by (9):

$$
\begin{gathered}
\min \left(\frac{\sigma_{\Delta p_{c}}^{2}}{\lambda_{c}^{2}}+\sigma_{\Delta \phi_{c}}^{2}+\sigma_{1, \Delta \text { iono }}^{2} I S F^{2}\right) \\
\left(\frac{m_{1}}{\lambda_{1}}+\frac{m_{2}}{\lambda_{2}}\right)-\left(\frac{n_{1}+n_{2}}{\lambda_{c}}\right)=0
\end{gathered}
$$

Obviously, (9) makes sure that the geometric elements, such as the geometric distance, tropospheric delay, clock errors and so on, can be offset in the cycle-slip calculation with (6). It means that the calculated cycle-slip combinations are free from the influences of geometric elements because of the geometry-free constraint by (9).

In the optimization model, the unknown parameters are 2 integer coefficients of the phase combination $m_{j}$, and 2 floating coefficients of the pseudorange combination $n_{j}$. The ergodic search method can be simply used to solve the optimization model. The searched range of integer coefficients is set as $[-10,10]$ because the absolute integers larger than 10 would result in larger combined noises in the phase combinations and should be excluded. Similarly, the searched range of floating coefficients is set as $[-1,1]$ with the step of 0.1 because the absolute floats larger than 1 would lead to larger combined noises in the pseudorange combinations and should be abandoned.

According to the optimization model (8) and the geometry-free constraint (9), 2 linearly independent phase combinations $\phi_{7, i}(i=1,2)$ are searched and shown in Table 1 , where $\phi_{7, i}=m_{i, 1} \phi_{1}+m_{i, 2} \phi_{2}$ is the phase combination; $m_{i, j}(i=1,2 ; j=1,2)$ are the coefficients of phase combination; $N_{7, i}$ is the corresponding ambiguity; $\lambda_{7, i}=c /\left(m_{i, 1} f_{1}+m_{i, 2} f_{2}\right)$ is the combined wavelength; $\sigma_{\phi_{7, i}}$ is the STD of in the phase combination; and ISFC $7, i$ (wrt B1, cycle) is the ISF in the phase combination.

Table 1. The optimized phase combinations of dual-frequency BDS.

\begin{tabular}{ccccc}
\hline$\phi_{7, i}$ & $m_{i, j}$ & $\lambda_{7, i}(\mathrm{~m})$ & $\sigma_{\phi_{7, i}}$ (Cycle) & ISFC $_{7, i}$ (wrt: B1, Cycle) \\
\hline$\phi_{7,1}$ & $4,-5$ & 1.4365 & 0.0640 & -12.8416 \\
$\phi_{7,2}$ & $-3,4$ & 2.0637 & 0.0500 & 11.3148 \\
\hline
\end{tabular}

Corresponding to $\phi_{7, i}$, the pseudorange combinations $p_{7, i}(i=1,2)$ are obtained according to the optimization model, as shown in Table 2, where $p_{7, i}=n_{i, 1} p_{1}+n_{i, 2} p_{2}$ is the pseudorange combination; $n_{i, j}(i=1,2 ; j=1,2)$ are the coefficients of pseudorange combination; $\sigma_{p_{7, i}}$ is the STD of the pseudorange combination; and $I S F P_{7, i}$ (wrt B1, m) is the ISF in the pseudorange combination. ISF $F_{7, i}=\operatorname{ISFC}_{7, i}+\operatorname{ISF} P_{7, i} / \lambda_{7, i}$ is the ISF of $N_{7, i}$ with the unit of the cycle; and $\sigma_{\Delta N_{7, i}}$ is the STD of $\Delta N_{7, i}$. 
Table 2. The optimized pseudorange combinations and cycle-slip combinations of dual-frequency BDS.

\begin{tabular}{cccccc}
\hline$p_{7, i}$ & $n_{i, j}$ & $\sigma_{p_{7, i}}(\mathbf{m})$ & ISFP $_{7, i}$ (wrt: B1, m) & ISF $_{7, i}$ (wrt: B1, Cycle) & $\sigma_{\Delta N_{7, i}}$ (Cycle) \\
\hline$p_{7,1}$ & $0.5,0.5$ & 0.2121 & 1.3362 & -11.9115 & 0.2343 \\
$p_{7,2}$ & $0.5,0.5$ & 0.2121 & 1.3362 & 11.9622 & 0.1710 \\
\hline
\end{tabular}

\subsection{Cycle Slip Solution}

Using the optimized phase combinations $\phi_{7, i}(i=1,2)$ and pseudorange combinations $p_{7, i}(i=1,2)$, the cycle-slip combination is calculated by:

$$
\Delta N_{7, i}=\frac{\Delta p_{7, i}}{\lambda_{7, i}}-\Delta \phi_{7, i}-I S F_{7, i} \Delta \text { iono }_{1, \mathrm{k}-1}
$$

According to (10), the floating values of $\Delta \mathrm{N}_{7, i}(i=1,2)$ and its covariance matrix can be obtained from the combinations of BDS dual-frequency phases and pseudoranges. Taking advantage of efficient performance in integer search, LAMBDA is utilized to seek the integer values of $\Delta \mathrm{N}_{7, i}$, with the inputs of the floating values of $\Delta \mathrm{N}_{7, i}(i=1,2)$ and its covariance matrix [23].

The cycle-slip combinations are the integer linear combination of the original cycle slips as:

$$
\left[\begin{array}{l}
\Delta N_{7,1} \\
\Delta N_{7,2}
\end{array}\right]=\left[\begin{array}{cc}
4 & -5 \\
-3 & 4
\end{array}\right]\left[\begin{array}{l}
\Delta N_{1} \\
\Delta N_{2}
\end{array}\right]
$$

where elements of the inverse matrix with respect to the matrix of combined coefficients in (11) still are integers. Hence, the original cycle slips can be obtained via the integer transformation from the cycle-slip combinations by:

$$
\left[\begin{array}{l}
\Delta N_{1} \\
\Delta N_{2}
\end{array}\right]=\left[\begin{array}{ll}
4 & 5 \\
3 & 4
\end{array}\right]\left[\begin{array}{l}
\Delta N_{7,1} \\
\Delta N_{7,2}
\end{array}\right]
$$

\subsection{Cycle Slip Discrimination against the Disturbance of Pseudorange Error}

Taking an insight into (10), the floating value of the cycle-slip combination is calculated from the pseudoranges. Unfortunately, the pseudorange error will contaminate the calculation of the cycle-slip combination. The BDS measurements, especially the pseudoranges, are easily affected by the observational environments and the quality of the observed signal. The noise level of the pseudorange is rather sensitive and large. Large pseudorange error will dramatically pollute the calculated value of the cycle-slip combination. As a result, the tremendous bias would be brought in the floating value of the cycle-slip combination, and the calculation of the cycle-slip combination becomes more sensitive to the observational environment and signal quality.

The precondition of correctly employing LAMBDA is that there is no significant bias in the inputted floating value. With the inputs of unbiased floating values and its covariance information, LAMBDA will output the integer values, which are closest to the inputted floating values, and a number of candidates sorted by the distance under the space defined by the covariance information. However, if there is a significant bias in the floating value of the cycle-slip combination, then the correct value of the cycle-slip combination is most likely to be placed in the candidates. Therefore, the disturbance from pseudorange error must be eliminated, and the correct value of the cycle-slip combination needs to be selected from all the candidates provided by LAMBDA, according to the inherent constraint of the BDS dual-frequency phases.

After correcting the ionospheric variation, epoch-difference GF is formed only with the dual-frequency phases and original cycle slips by:

$$
\Delta L_{4}=\left(\Delta \phi_{1}+\Delta N_{1}\right) \lambda_{1}-\left(\Delta \phi_{2}+\Delta N_{2}\right) \lambda_{2}+\left(1-f_{1}^{2} / f_{2}^{2}\right) \Delta \text { iono }_{1, k-1}
$$


In the epoch-difference GF, after correcting the ionospheric variation, the ionospheric effect and the geometric elements have all been counteracted. The phase bias and the multipath effect are nearly constant in a short time and could be offset by the epochdifference treatment. Therefore, if the fixed values of the original cycle slips $\Delta N_{j}$ are correct, the value of epoch-difference GF should approach 0 as:

$$
\Delta L_{4} \rightarrow 0
$$

The original cycle slip $\Delta N_{j}$ can be calculated from Equations (10) and (12). Then, $\Delta L_{4}$ it is obtained by substituting $\Delta N_{\mathrm{j}}$ into (13). LAMBDA can provide several candidate sets of the cycle-slip combinations $\Delta \mathrm{N}_{7, i}(n)$, where $\mathrm{n}$ is the index on the candidate sets of the cycle-slip combinations. The candidates of the original cycle slips $\Delta N_{\mathrm{j}}(\mathrm{n})$ are transformed from $\Delta \mathrm{N}_{7, i}(n)$ by (12) as

$$
\left[\begin{array}{l}
\Delta N_{1}(\mathrm{n}) \\
\Delta N_{2}(\mathrm{n})
\end{array}\right]=\left[\begin{array}{ll}
4 & 5 \\
3 & 4
\end{array}\right]\left[\begin{array}{l}
\Delta N_{7,1}(\mathrm{n}) \\
\Delta N_{7,2}(\mathrm{n})
\end{array}\right]
$$

Correspondingly, values of $\Delta L_{4}(\mathrm{n})$ are calculated by (13) with the candidates of the original cycle slip $\Delta N_{\mathrm{j}}(\mathrm{n})$ as:

$$
\Delta L_{4}(\mathrm{n})=\left(\Delta \phi_{1}+\Delta N_{1}(\mathrm{n})\right) \lambda_{1}-\left(\Delta \phi_{2}+\Delta N_{2}(\mathrm{n})\right) \lambda_{2}+\left(1-f_{1}^{2} / f_{2}^{2}\right) \Delta \text { iono }_{1, k-1}
$$

According to the characteristic that the epoch-difference GF after correcting the ionospheric variation approaches to 0 , the discrimination function is proposed to select the final cycle-slip value by:

$$
\min \left(\left|\Delta L_{4}(\mathrm{n})\right|\right)
$$

where the cycle slips resulting in the minimum of absolute epoch-difference GF after correcting the ionospheric variation are selected as the final cycle slips. It should be noticed that the cycle slips of $(590,763)$ must be excluded from the candidates of the original cycle slips. Because the discrimination value resulted from the candidate cycle slips of $(590,763)$ is 0 , the candidate original cycle slips of $(590,763)$ may provide a fake appeal and spurious guidance to the discrimination function.

Summarily, the process flow diagram is shown in Figure 2. The implemented steps are following: (1) GFCSD is employed to find the first epoch of phase data without cycle slip. (2) The ionospheric variation at the first epoch is calculated by Equation (4) with the epoch-differenced phases. (3) The ionospheric variation at the next epoch is corrected with that of at the previous epoch. (4) After correcting the ionospheric variation, floats of the cycle-slip combinations and their covariance are calculated with Equation (10). (5) The candidates of the cycle-slip combinations are supplied from the LAMBDA with the inputs of floats of the cycle-slip combinations and their covariance. (6) The candidates of the original cycle slips are transformed from the candidates of the cycle-slip combinations with (12). (7) The discrimination values that are the epoch-difference GF after correcting the ionospheric variation can be calculated by (16) with the candidates of the original cycle slips. (8) The ranking is enforced on the absolute discrimination values according to (17). (9) Finally, the values of the cycle slips lead to the minimum of absolute discrimination values are outputted as the proposal values of the cycle slips. 


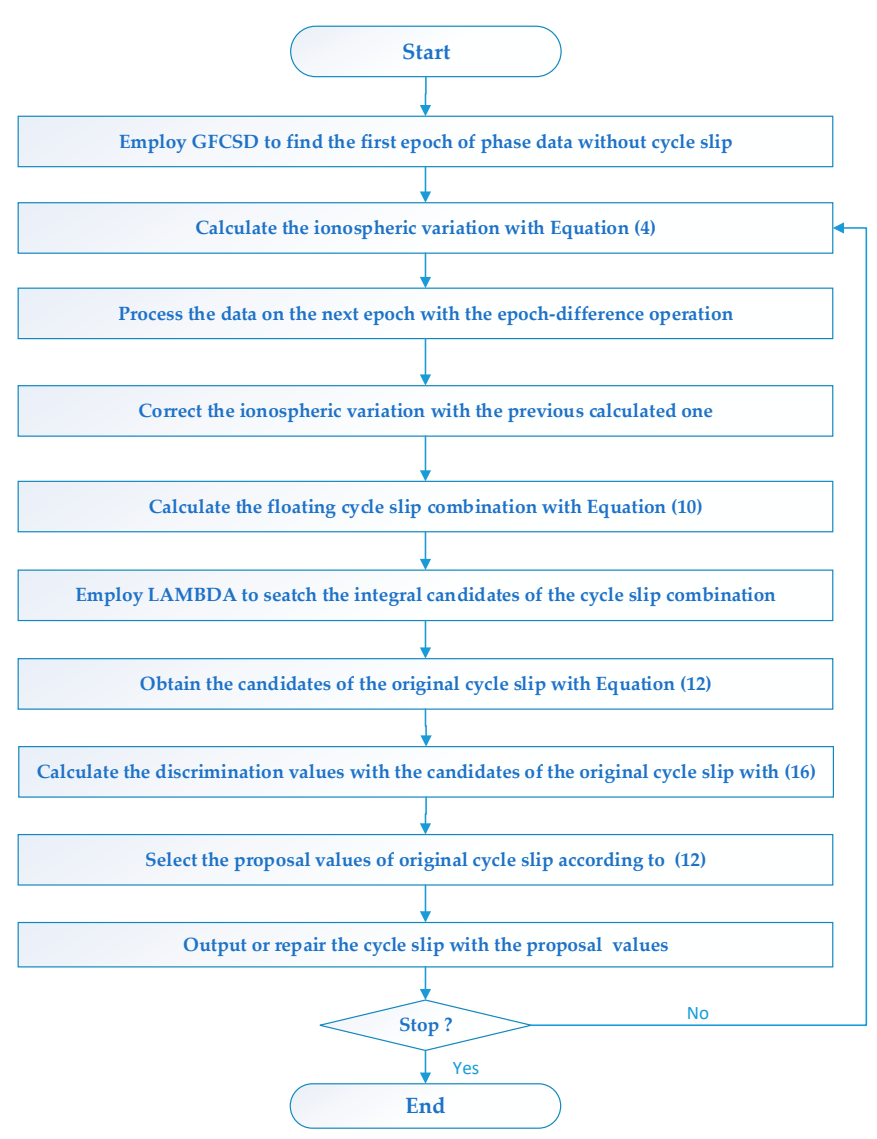

Figure 2. The process flow of the cycle-slip repair (CSR) method for BDS dual-frequency phases (BDCSR).

\section{Test Description}

Comparative tests were designed to analyze and verify the results of the BDCSR methodology. On one hand, some extreme conditions were chosen in the experiments. During the period of severe ionospheric variation under the strong geomagnetic storm, the CUT0 station in the low latitude area with active ionosphere was selected to collect the BDS dual-frequency observation data. In addition, some representative experiments were deployed to compare and validate the repair performance.

The real BDS dual-frequency data were collected from stations at high and low latitudes shown in Table 3, with the sampling interval of $30 \mathrm{~s}$ starting from UTC 2016/05/08 00:00:00. On that day, a geomagnetic storm occurred with a level bigger than 7 , and the values of the Kp index ranged from 67 to 94 . During the geomagnetic storm, remarkable ionospheric variation was presented in the active ionosphere with respect to the quiet ionosphere. Since CUT0 can observe more BDS satellites, the CSR results from different satellites at the CUT0 are plotted and shown in detail in the following Sections.

Table 3. The conditions of different collected stations.

\begin{tabular}{ccccc}
\hline Station Name & Latitude & Longitude & Receiver Type & Antenna Type \\
\hline CUT0 & -32.004 & 115.895 & TRIMBLE & TRM59800.00 \\
ANMG & 2.785 & 101.507 & $\begin{array}{c}\text { TRIMBLE } \\
\text { NETR9 }\end{array}$ & JAVRINGANT_DM \\
RGDG & -53.786 & -67.752 & TRIMBLE & TRM59800.00 \\
KIRU & 67.857 & 20.968 & SEPT POLARX5 & SEPCHOKE_B3E6 \\
\hline
\end{tabular}


BDS includes GEO, IGSO, and MEO satellites. The GEO Satellite keeps being stationary relative to the Earth, and the ionospheric delay may change smoothly. However, the MEO and IGSO satellites move fast to the Earth, and the ionospheric delay varies greatly between epochs. Therefore, comparison experiments are arranged with different dynamic conditions between GEO Satellites and MEO satellites under the environment of severe ionospheric variation during the geomagnetic storm. The situations of IGSO are similar to those of MEO.

Since the GEO Satellite maintains a medium elevation to the station, the better quality of pseudoranges is ensured from the GEO observations. However, the elevations of MEO and IGSO are different from that of the GEO. There is a rising or falling window for the MEO satellite to the station, and a low elevation will result in high-level noises of pseudoranges. Therefore, comparison experiments are designed with different elevation spans from the GEO Satellite and MEO satellite during the geomagnetic storm.

Obviously, the bias close to or over 0.5 cycles will cause the fix failure of directly rounding the float of the cycle-slip combination. Therefore, half of the combined wavelength is selected as the upper limit of the epoch-difference pseudorange error, and the CSR test under the influence of gross error on real pseudorange is planned. A gross error of $0.7 \mathrm{~m}$ is added to the real epoch-difference pseudoranges epoch-by-epoch, and the real data with a large pseudorange error is obtained. Under the condition of real pseudorange adding gross error, results of the BDCSR methodology and the conventional CSR with the optimized cycle-slip combinations are compared.

The small cycle slips are hard to be detected and repaired. In addition, the special cycle slips make some CSD methods to be invalid. Thereby, most studies take the small and special cycle slips to examine the performances of CSR or CSD. In this study, the small cycle slips $(1,0)$ and special cycle slips $(1,1)$ are added to the phase data without cycle slip, and the performance of the BDCSR method is evaluated. Actually, for the phase data without cycle slip, all the cycle-slip values are 0 , so the correct results of CSR in the time sequences should be a straight line with the $\Delta \mathrm{N}_{j}=0$ in Figures. Similarly, for the phase data without cycle slip, the known cycle slips $(1,0)$ are added to the epoch-difference phases epoch-by-epoch. All the cycle-slip values are $(1,0)$. Therefore, the correct results of CSR in the time sequences should be a straight line with the $\Delta \mathrm{N}_{1}=1$ and a straight line with $\Delta \mathrm{N}_{2}=0$.

In order to examine the validity and reliability of the BDCSR methodology, the following compared schemes are designed and listed in Table 4. Scheme 1: BDCSR employs the $\Delta N_{7, i}(i=1,2)$ after correcting the ionospheric variation to calculate the cycle-slip combinations and utilizes the discrimination function (17) to select the final cycle slips. Then, the results after the selection with the discrimination function are provided from the BDCSR methodology. Scheme 2: In the absence of the discrimination function, the conventional dual-frequency CSR (CDCSR) only adopts the optimized cycle-slip combinations $\Delta N_{7, i}$ $(i=1,2)$ after correcting the ionospheric variation to directly fix the cycle slip. Then, the results before the selection are provided from CDCSR. Scheme 3: CSR, without correcting ionospheric variation (WICSR), utilizes the $\Delta N_{7, i}(i=1,2)$ by treating the ionospheric variation as 0 to repair the cycle slip. Scheme 4: CSD with traditional MW (MWCSD) is used to perform cycle-slip detection. Finally, the results of the BDCSR methodology, CDCSR, WICSR and MWCSD are compared, and the validity and reliability of the BDCSR method are verified carefully.

Table 4. The comparison of different testing methods.

\begin{tabular}{ccccc}
\hline $\begin{array}{c}\text { Compared } \\
\text { Methods }\end{array}$ & $\begin{array}{c}\text { MW Cycle-Slip } \\
\text { Combination }\end{array}$ & $\begin{array}{c}\text { Optimized Cycle-Slip } \\
\text { Combination }\end{array}$ & $\begin{array}{c}\text { Correction the Ionospheric } \\
\text { Variation }\end{array}$ & $\begin{array}{c}\text { Selection with the } \\
\text { Discrimination Function }\end{array}$ \\
\hline MWCSD & $\checkmark$ & & & \\
WICSR & & $\checkmark$ & $\checkmark$ & \\
CDCSR & & $\checkmark$ & $\checkmark$ & $\checkmark$ \\
BDCSR (proposed) & & $\checkmark$ & \\
\hline
\end{tabular}




\section{Results and Discussion}

Experiments described in Section 3 were implemented. After comparing the experimental results, some benefits from this study were validated. First, the results of the BDCSR method were compared with those of WICSR. The influences of severe ionospheric variations on calculating the cycle-slip combinations of the GEO Satellites and MEO satellites were compared from different dynamic conditions with respect to the stations. The actual correction precision of ionospheric variation was investigated under the highly active ionospheric circumstance during a geomagnetic storm. Second, results of the BDCSR method were compared with those of CDCSR. In cases of the fine condition with medium elevations, the weak condition with low elevations, and the bad condition of adding gross error on the real epoch-difference pseudoranges, the performance of the BDCSR method against the disturbance of large pseudorange error was validated. Finally, in situations of presenting small cycle slips and special cycle slips, results of the BDCSR method were compared with those of MWCSD.

\subsection{The Influence of Severe Ionospheric Variation on Low-Dynamic GEO Satellite}

The tests obtained BDS dual-frequency observations with a sampling interval of $30 \mathrm{~s}$ at the CUT0 station in the low latitudes. During the period with the 7-level geomagnetic storm, the observation data were processed. Before correcting ionospheric variation, cycleslip combinations of WICSR can be obtained from the phase data without cycle slip. In contrast, BDCSR provided the cycle-slip combinations after correcting the ionospheric variation. Cycle-slip combinations of WICSR and BDCSR from the GEO Satellite 2 are shown in Figure 3. Figure 3 displays that cycle-slip combinations from the BDCSR methodology are almost within -0.5 to 0.5 cycles and distribute around 0 consistently. However, cycle-slip combinations of WICSR are slightly biased from 0 with respect to those of the BDCSR methodology.
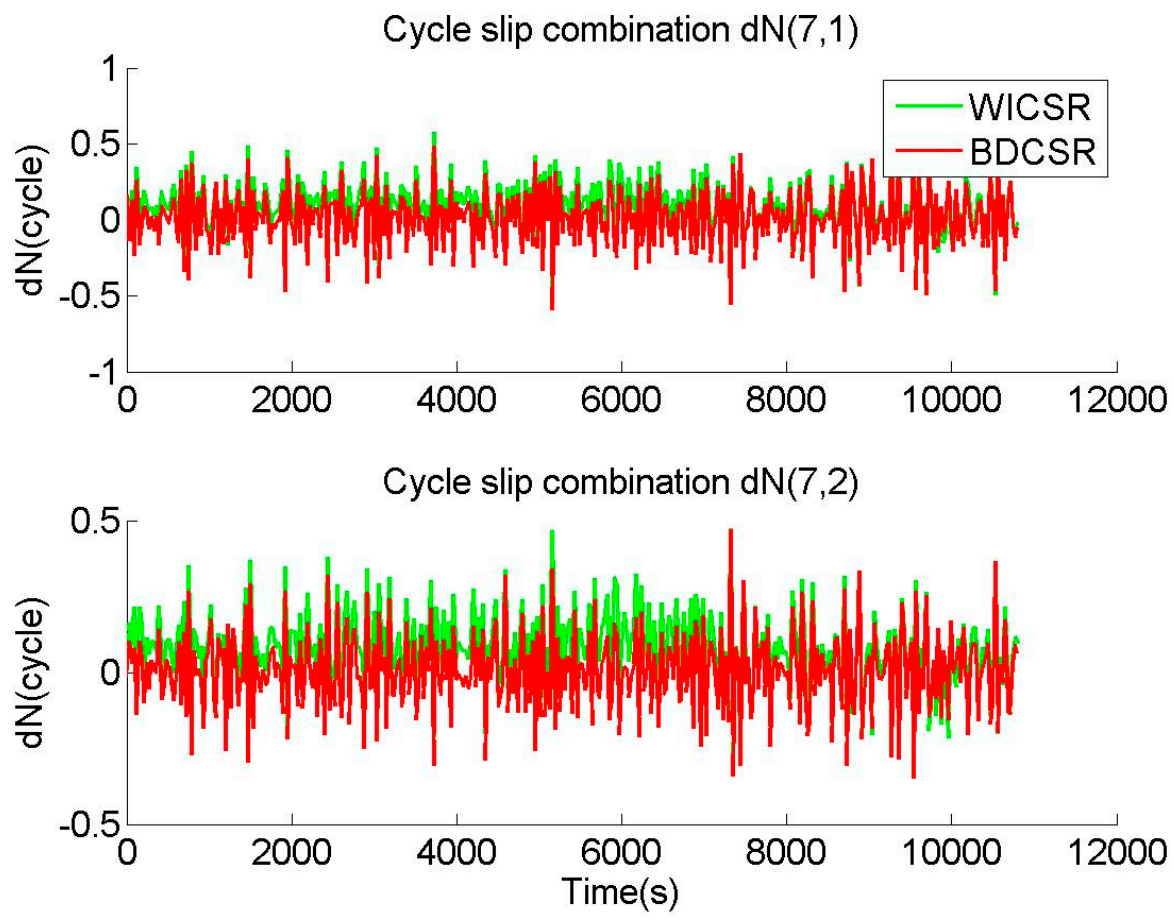

Figure 3. The cycle-slip combinations of WICSR without correcting the ionospheric variations and those of the BDCSR methodology after correcting the ionospheric variations, where the BDS dualfrequency pseudoranges and phases without cycle slip were collected from the GEO Satellite 2 during the geomagnetic storm, started from UTC 8 May 2016 00:00:00 with an interval of $30 \mathrm{~s}$ at the CUT0 station. 
The original cycle slips of WICSR and BDCSR are shown in Figure 4. Results of WICSR in Figure 4 show that there are two mistakes that were present in the WICSR on the epochs of $3750 \mathrm{~s}$ and $7200 \mathrm{~s}$. The reason is that the absolute values of $\Delta \mathrm{N}_{7,1}$ WICSR shown in Figure 3 are a little larger than 0.5 cycles on the corresponding epochs. It indicates that the application of WICSR to the GEO Satellite receives tiny influence from the ionospheric variation by reason of the GEO Satellite being almost stationary relative to the station. However, the correction of the ionospheric variation on the GEO Satellite is still necessary.
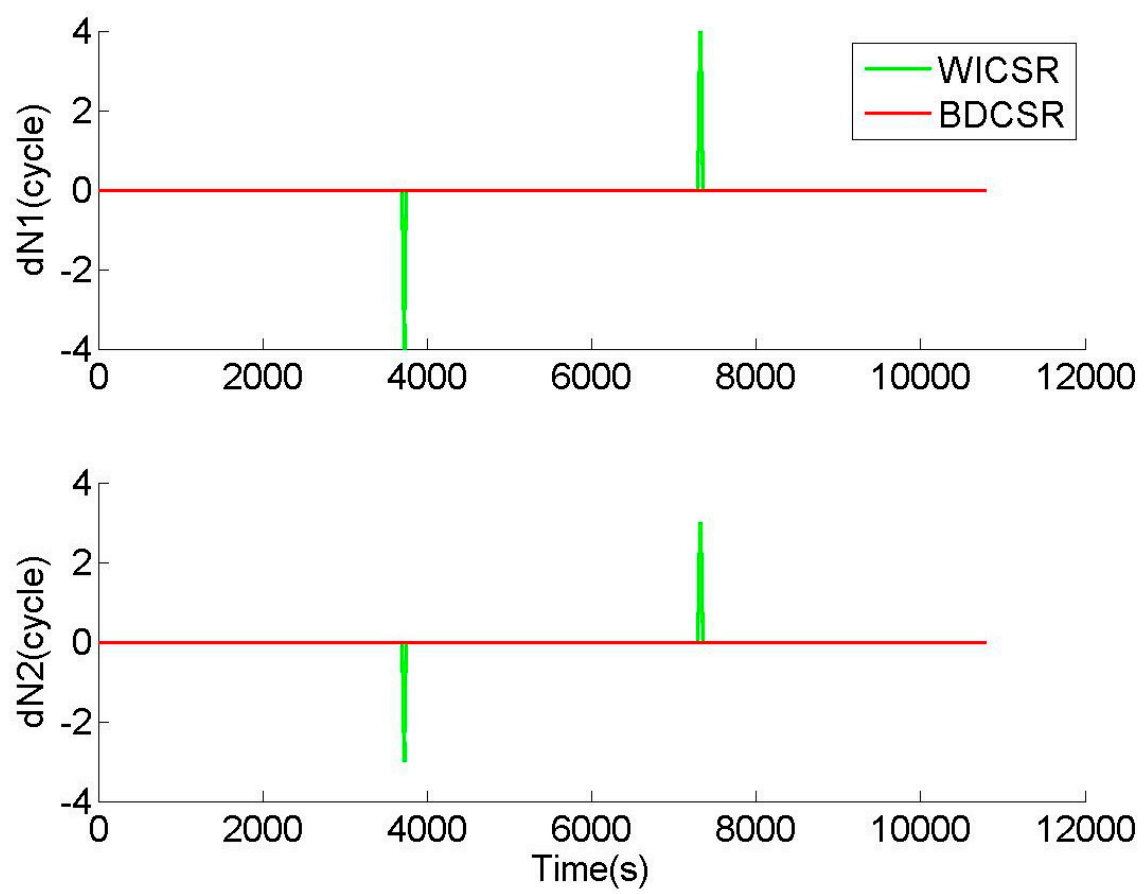

Figure 4. The original cycle slips without correcting the ionospheric variations (WICSR) and those of the BDCSR methodology after correcting the ionospheric variations, where the BDS dualfrequency pseudoranges and phases without cycle slip were collected from the GEO Satellite 2 during the geomagnetic storm, started from UTC 8 May 2016 00:00:00 with an interval of $30 \mathrm{~s}$ at the CUT0 station.

4.2. The Influence of Severe Ionospheric Variation on the High-Dynamic MEO and IGSO Satellites and Its Correction Performance

The cycle-slip combinations $\Delta \mathrm{N}_{7, i}(i=1,2)$ can be calculated with the optimized phase combinations $\phi_{7, i}$ and the pseudorange combinations $p_{7, i}$ from dual-frequency BDS. In the case of phase data without cycle slip, Figure 5 shows the sequence of the cycle-slip combinations on MEO Satellite 11 provided by WICSR without correcting the ionospheric variation and BDCSR after correcting the ionospheric variation. In addition, the original cycle slips of the BDCSR methodology and WICSR from MEO Satellite 11 are displayed in Figure 6. 

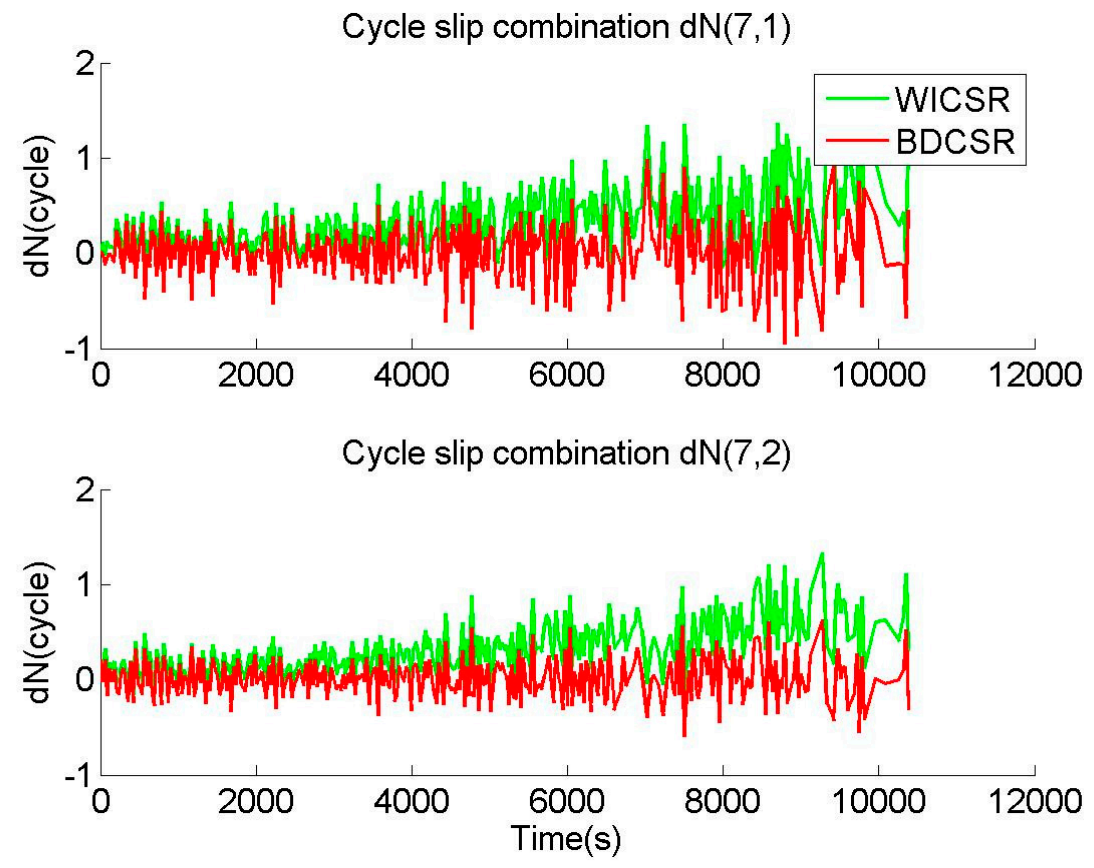

Figure 5. The cycle-slip combinations of WICSR without correcting the ionospheric variations and those of the BDCSR methodology after correcting the ionospheric variations, where the BDS dualfrequency pseudoranges and phases without cycle slip were collected from the MEO Satellite 11 during the geomagnetic storm, started from UTC 8 May 2016 00:00:00 with an interval of $30 \mathrm{~s}$ at the CUT0 station.
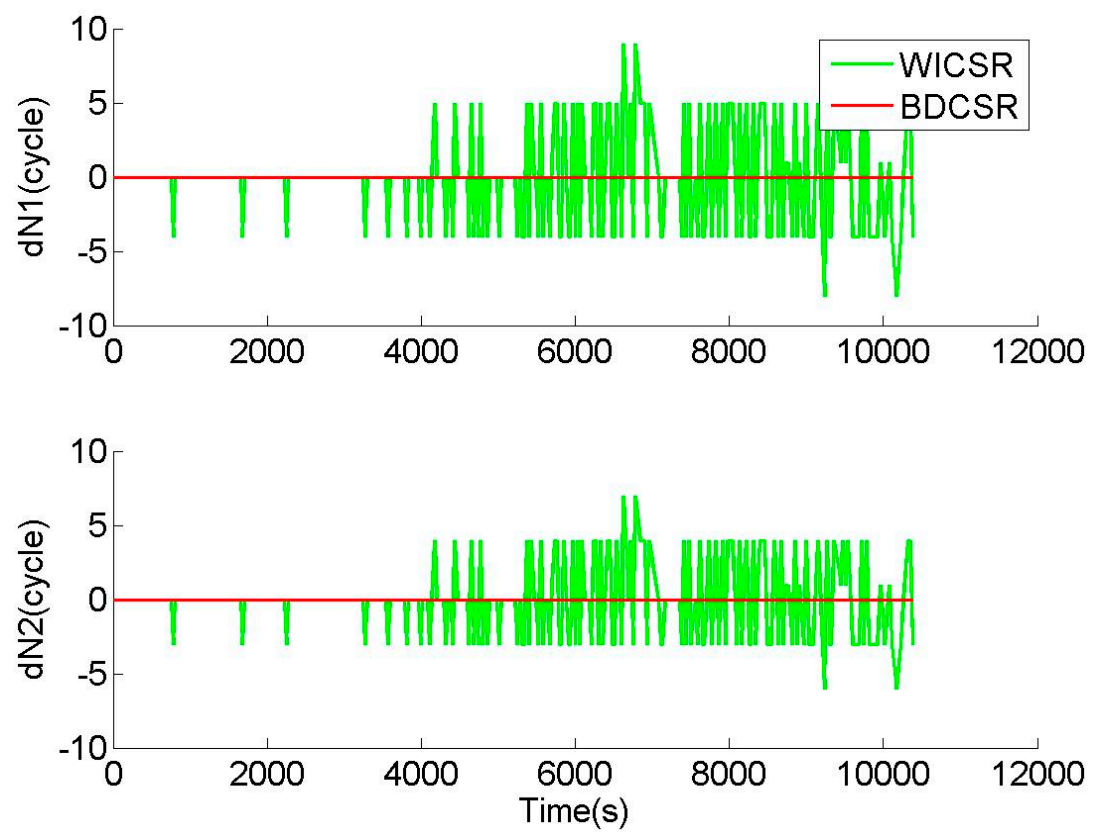

Figure 6. The original cycle slips of WICSR without correcting the ionospheric variations and those of the BDCSR methodology after correcting the ionospheric variations, where the BDS dualfrequency pseudoranges and phases without cycle slip were collected from the MEO Satellite 11 during the geomagnetic storm, started from UTC 8 May 2016 00:00:00 with an interval of $30 \mathrm{~s}$ at the CUT0 station.

Figure 5 displays that after $4000 \mathrm{~s}$, the absolute $\Delta \mathrm{N}_{7, i}$ from WICSR appears an increasing trend and gradually exceeds 0.5 to 1.8 . The float deviating from the correct integer greater than 0.5 may cause the float to be fixed to the incorrect integer. Figure 1 shows that 
the ionospheric variations from MEO Satellite 11 after $4000 \mathrm{~s}$ present a visible increasing procedure from 0.02 to 0.08 . Table 2 shows the max ISF in the cycle-slip combinations is 11.96. Then, the influence of ionospheric variation on the cycle-slip combination of MEO Satellite 11 is $0.24-0.96$ cycles. Clearly, the bias resulted from the ionospheric variation as large as 0.96 is disastrous and unbearable. It implies that the value of the cycle-slip combination is gravely polluted by the ionospheric variation, and the CSR failures will come up with the WICSR.

As shown in Figure 5, when the BDCSR methodology has corrected the ionospheric variation, the absolute values of the cycle-slip combinations $\Delta \mathrm{N}_{7, i}(i=1,2)$ provided by the BDCSR methodology from MEO Satellite 11 are obviously smaller than those of WICSR. It confirms that after correcting the ionospheric variation, the notable tendency in the cycle-slip combination resulted from the influence of the ionospheric variation was distinctly mitigated, and a more beneficial condition is prepared to determine and repair the cycle slip.

The original cycle slips determined by the BDCSR methodology in Figure 6 show that all results of the BDCSR methodology are zero; it is accordant with the actual situation of phase data without cycle slip. However, many results of WICSR are wrong since the original cycle slips outputted from WICSR are nonzero. Figure 7 shows the original cycle slips of WICSR and BDCSR from the IGSO Satellite 6. The same situation is demonstrated that WICSR confronts many mistakes because of the significant influence of ionospheric variation.
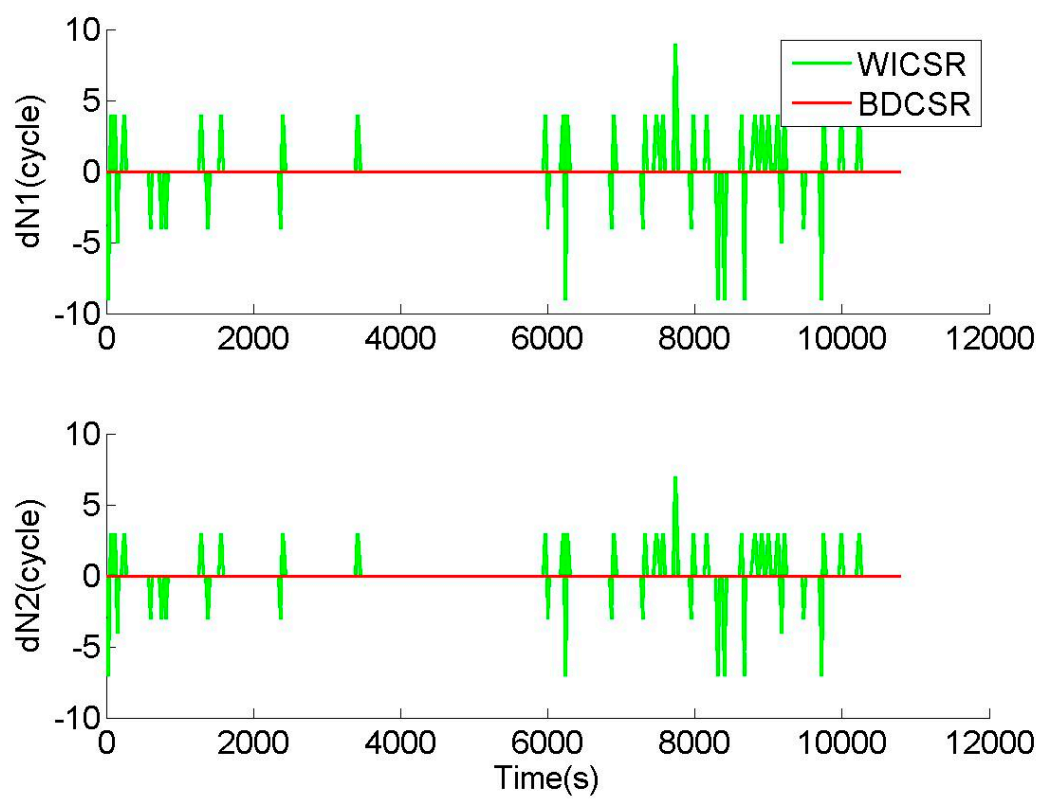

Figure 7. The original cycle slips of WICSR without correcting the ionospheric variations and those of the BDCSR methodology after correcting the ionospheric variations, where the BDS dualfrequency pseudoranges and phases without cycle slip were collected from the IGSO Satellite 6 during the geomagnetic storm, started from UTC 8 May 2016 00:00:00 with an interval of $30 \mathrm{~s}$ at the CUT0 station.

\subsection{CSR Results of the GEO Satellite at Medium Elevation during a Geomagnetic Storm}

The GEO Satellites present a fine observational condition to the Earth since not only a medium elevation is formed to the stations at low and middle latitudes, but also the elevations of the GEO Satellite are unchanged. Thus, the high-quality observation data can be obtained from the GEO Satellites with relatively stable and medium elevations. In the case of phase data without cycle slip from the GEO Satellite under fine observational conditions, CSR experiments were carried out to verify the performances of the BDCSR methodology and CDCSR. 
According to (15) and (16), the discrimination value is the absolute epoch-difference GF calculated with the original cycle slips after correcting the ionospheric variation. Before the selection with the discrimination function, CDCSR directly adopts the outputs of the cycle-slip fixation. After the selection with the discrimination function, BDCSR obtains the final cycle slip. Using the cycle-slip values provided by the BDCSR methodology, the discrimination value after the selection can be calculated. With the cycle-slip values supplied by CDCSR, the discrimination value before the selection can be obtained. Then, the ratio is calculated from the discrimination value of CDCSR, dividing that of the BDCSR methodology. The larger the ratio value is, the more sensitive the discrimination function is, and the more prominent the ability to distinguish the correct cycle slips is. BDCSR and CDCSR processed the phase data without cycle slip under the fine condition with the medium elevations. The cycle-slip values of CDCSR before selection, those of the BDCSR methodology after selection, and the ratios are shown in Figure 8.
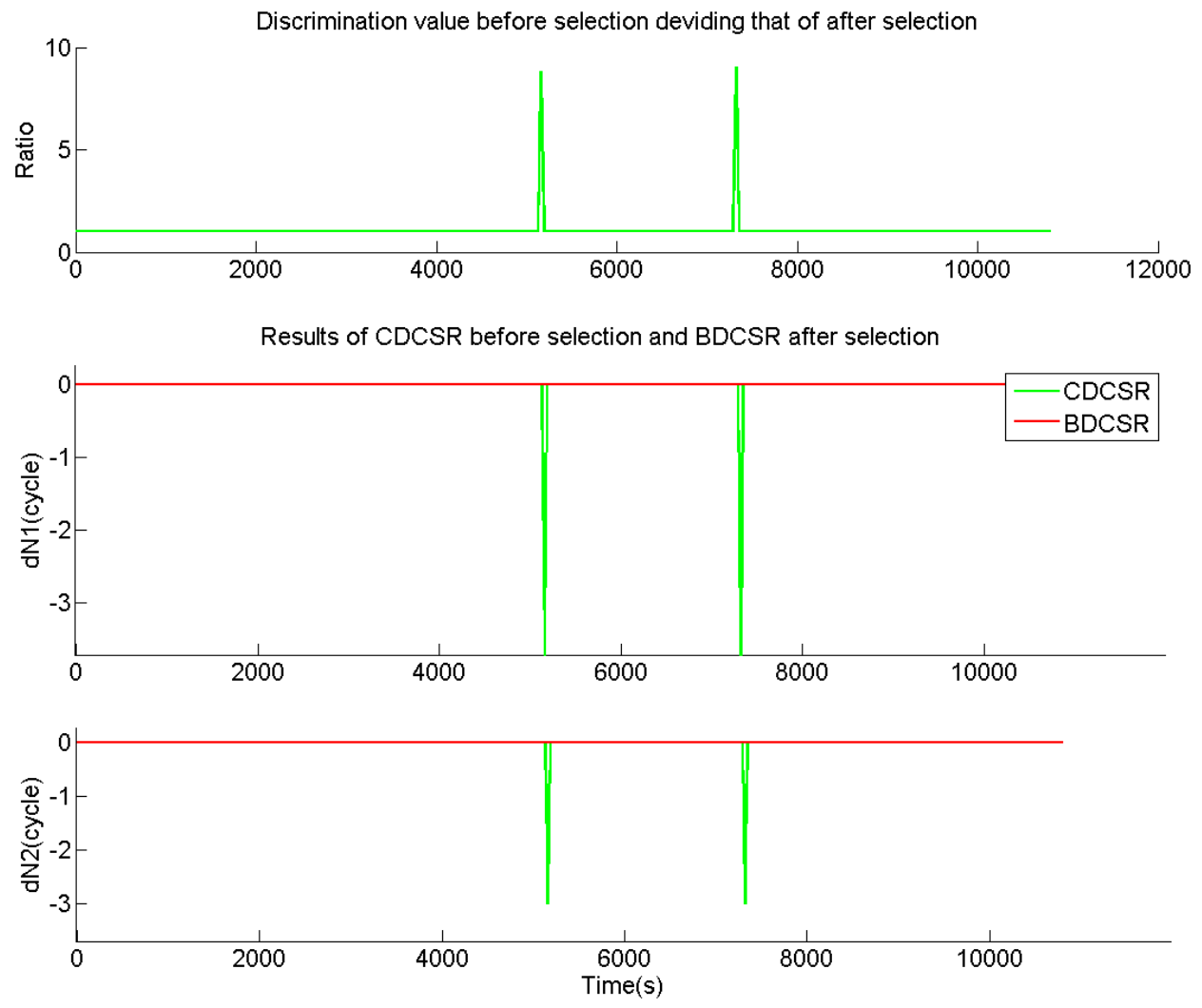

Figure 8. Results of CDCSR before selection and BDCSR after selection with the discrimination function from the GEO Satellite 2 under a fine condition with the medium elevations during the geomagnetic storm, where the phase data without cycle slip was collected at the CUT0 station started from UTC 8 May 2016 00:00:00 with an interval of $30 \mathrm{~s}$.

In the fine observational condition with the medium elevations, Figure 8 shows that the cycle-slip values of CDCSR before selection with the discrimination function contain two wrong results in the phase data without cycle slip. The wrong results are located at $5100 \mathrm{~s}$ and $7200 \mathrm{~s}$. Since the cycle-slip combinations of CDCSR are the same as those of the BDCSR methodology, the floating values of the cycle-slip combination of the BDCSR methodology can be analyzed from Figure 3; it is found that the absolute value of the cycle-slip combination at $5100 \mathrm{~s}$ and $7200 \mathrm{~s}$ is slightly larger than 0.5 cycles. Although the elevations of the GEO Satellite were maintained at about 37 degrees, there are still existed occasional large pseudorange errors. The large pseudorange error leads to a remarkable bias in the calculated value of the cycle-slip combination. When the absolute bias exceeds 0.5 , the fixed result is most likely to be polluted. In this case, CSR failures would be caused by applying the directly fixed value. 
In Figure 8, cycle-slip values of the BDCSR methodology after selection with the discrimination function shows that all cycle-slip values of the BDCSR methodology are 0 , and results of the BDCSR methodology conform with the situation of phase data without cycle slip. It supports that the cycle-slip values determined by the discrimination function are correct. Although some absolute floats of the cycle-slip combination exceed 0.5 , the determination of the cycle slip is not disturbed by the large bias with the help of the discrimination function.

Let us take an insight into the ratios from the discrimination value of CDCSR dividing that of the BDCSR methodology. In Figure 8, the ratios increased greatly on the epochs of $5100 \mathrm{~s}$ and $7200 \mathrm{~s}$, where the CDCSR failed to repair the cycle slips in the absence of selecting with the discrimination function. When the CDCSR successfully repaired cycle slips, the ratios are all 1. It implies that once the cycle slips are selected and determined correctly, the absolute value of the epoch-difference GF after correcting the ionospheric variation turns to be the smallest. Therefore, the correct cycle-slip values can be determined according to the discrimination function to select the cycle-slip candidates resulted in the minimum absolute value of epoch-difference GF after correcting the ionospheric variation.

\subsection{CSR Results of MEO and IGSO Satellites at Low Elevations during a Geomagnetic Storm}

The MEO and IGSO satellites not only move fast relative to the station but also the scope of MEO and IGSO satellites cover the low-elevation condition in the rising and falling courses. During the low elevations, the observational noises are larger, so this situation will be a challenging environment for CSR. In the experiment, the observation data of the MEO Satellite 11 is selected because the elevations of MEO Satellite 11 falls from 45 degrees to 5 degrees and cover a completely falling course. For the phase data without cycle slip, comparisons of the BDCSR methodology and CDCSR were carried out, the role of the discrimination function was verified in aspects of eliminating the disturbance of pseudorange error and ensuring the reliability of CSR.

Under the weak condition of low elevations, BDCSR and CDCSR processed the phase data without cycle slip. The cycle-slip values of CDCSR before selection with the discrimination function and those of the BDCSR methodology after the selection and the ratios from the MEO Satellite 11 are shown in Figure 9. The floats of corresponding cycleslip combinations can be seen in Figure 5. The floats of the MW cycle-slip combinations are shown in Figure 10. In addition, the results of the BDCSR methodology and CDCSR from the IGSO Satellite 6 are shown in Figure 11.

Figure 9 shows the cycle-slip values of CDCSR and BDCSR from the MEO Satellite 11, with the elevations descending from 45 degrees to 5 degrees. In the case of phase data without cycle slip, there are a large number of incorrect cycle-slip values existed in the results of CDCSR. CDCSR and BDCSR adopt the same cycle-slip combinations shown in Figure 5. During the period after $6000 \mathrm{~s}$, the elevations fall from 22 degrees to 5 degrees. At this time, the results of CDCSR are incorrect on most epochs. Figure 5 shows that the influence of pseudorange error begins to increase radically. The absolute floats of the calculated cycle-slip combinations have increasingly deviated from the true values of more than 0.5 cycles. At this moment, CDCSR applied the directly fixed cycle-slip values, and many repair failures happened. In Figure 9, the ratios are greatly increased when the wrongly repaired values are confronted by the CDCSR before the selection via the discrimination function. While the results of the BDCSR methodology after selection with the discrimination function are correct, the cycle-slip values of the BDCSR methodology are 0 , which is in accordance with the real situation of the phase data without cycle slip. The reason is that the discrimination function exerts a reliable ability to recognize the correct cycle slips. 

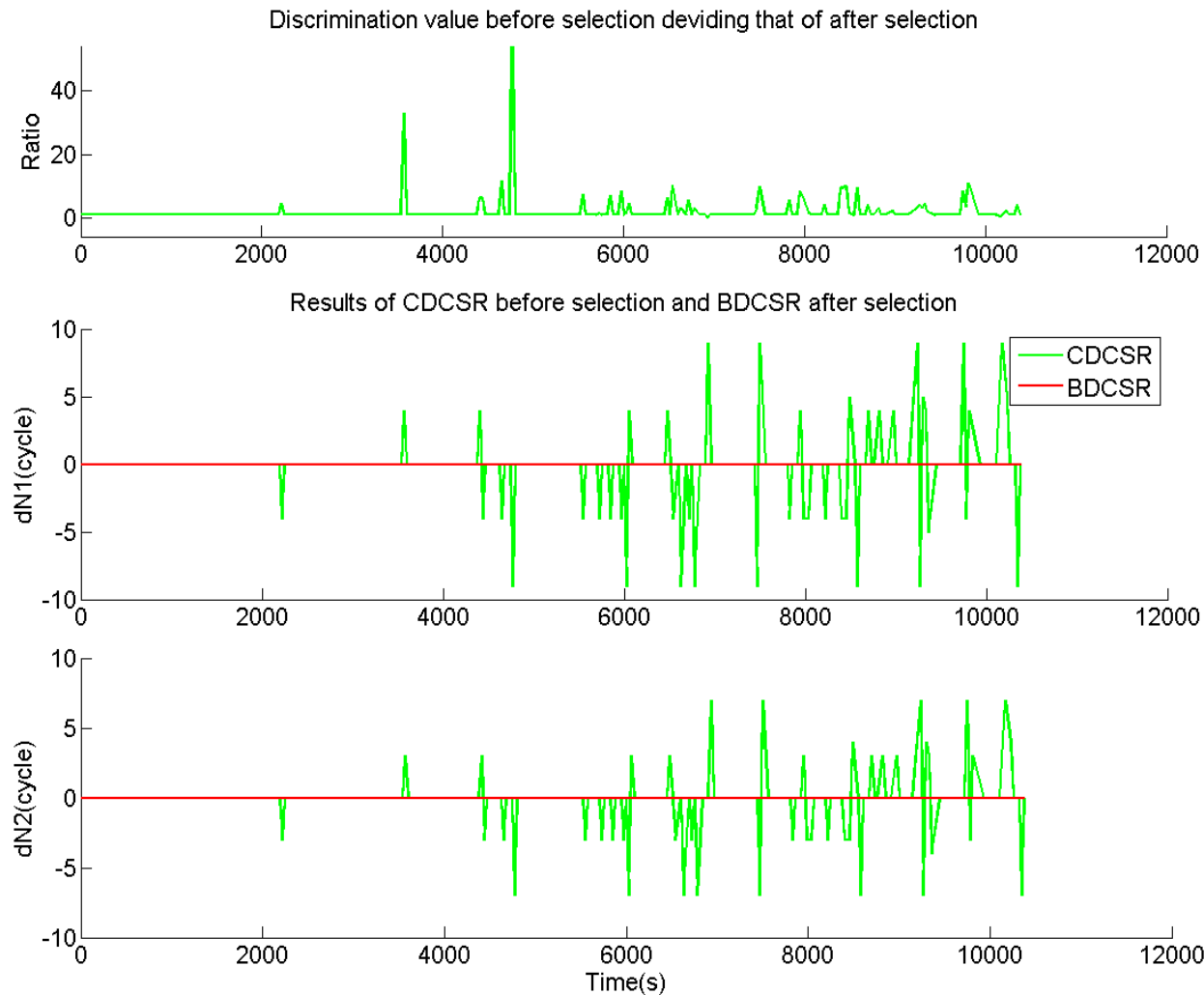

Figure 9. Results of CDCSR before selection and BDCSR after selection with the discrimination function from MEO Satellite 11 in a weak condition of low elevations during the geomagnetic storm, where the phase data without cycle slip was collected at the CUT0 station started from UTC 8 May 2016 00:00:00 with an interval of $30 \mathrm{~s}$.

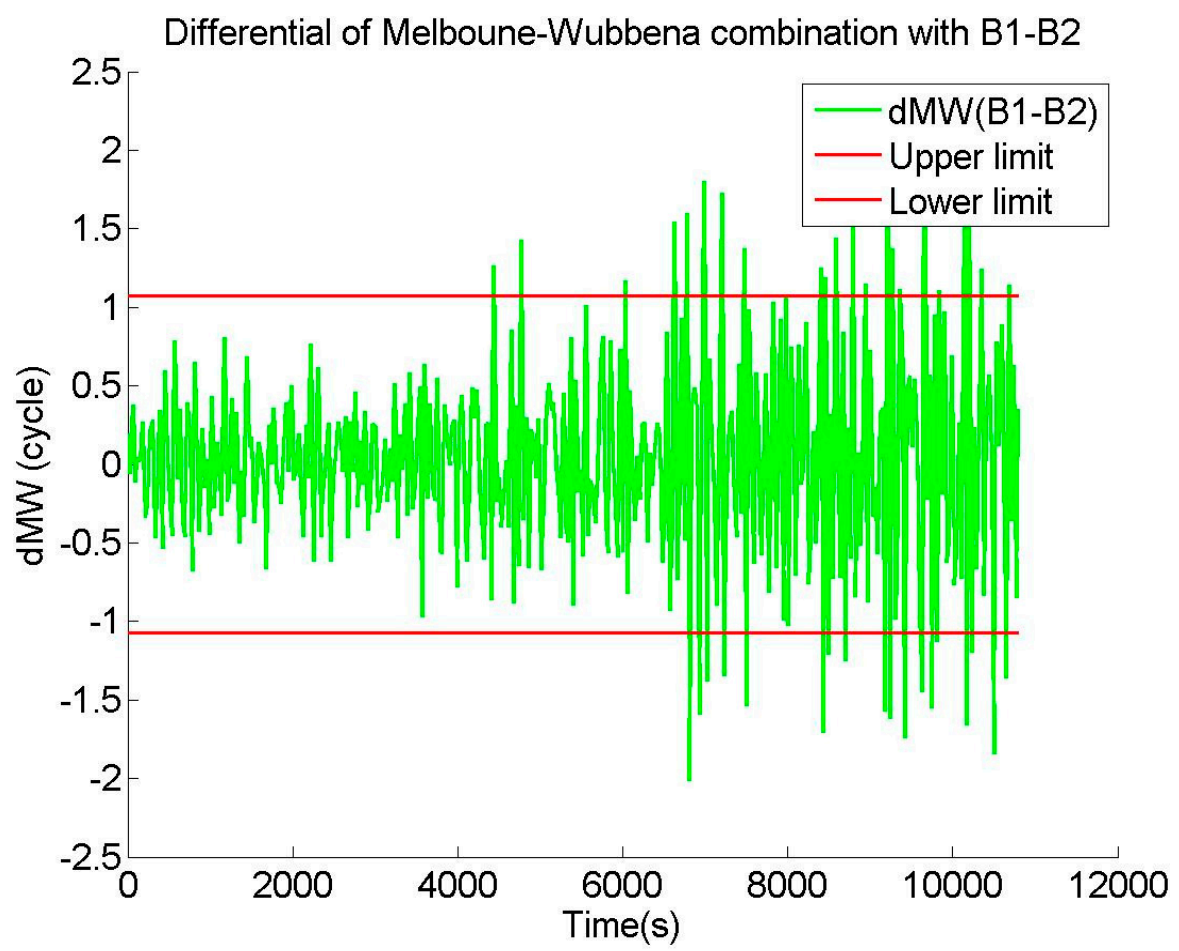

Figure 10. Sequences of the MW cycle-slip combinations from MEO Satellite 11 in a weak condition of low elevation during the geomagnetic storm, where the phase data without cycle slip was collected at the CUT0 station started from UTC 8 May 2016 00:00:00 with an interval of $30 \mathrm{~s}$. 

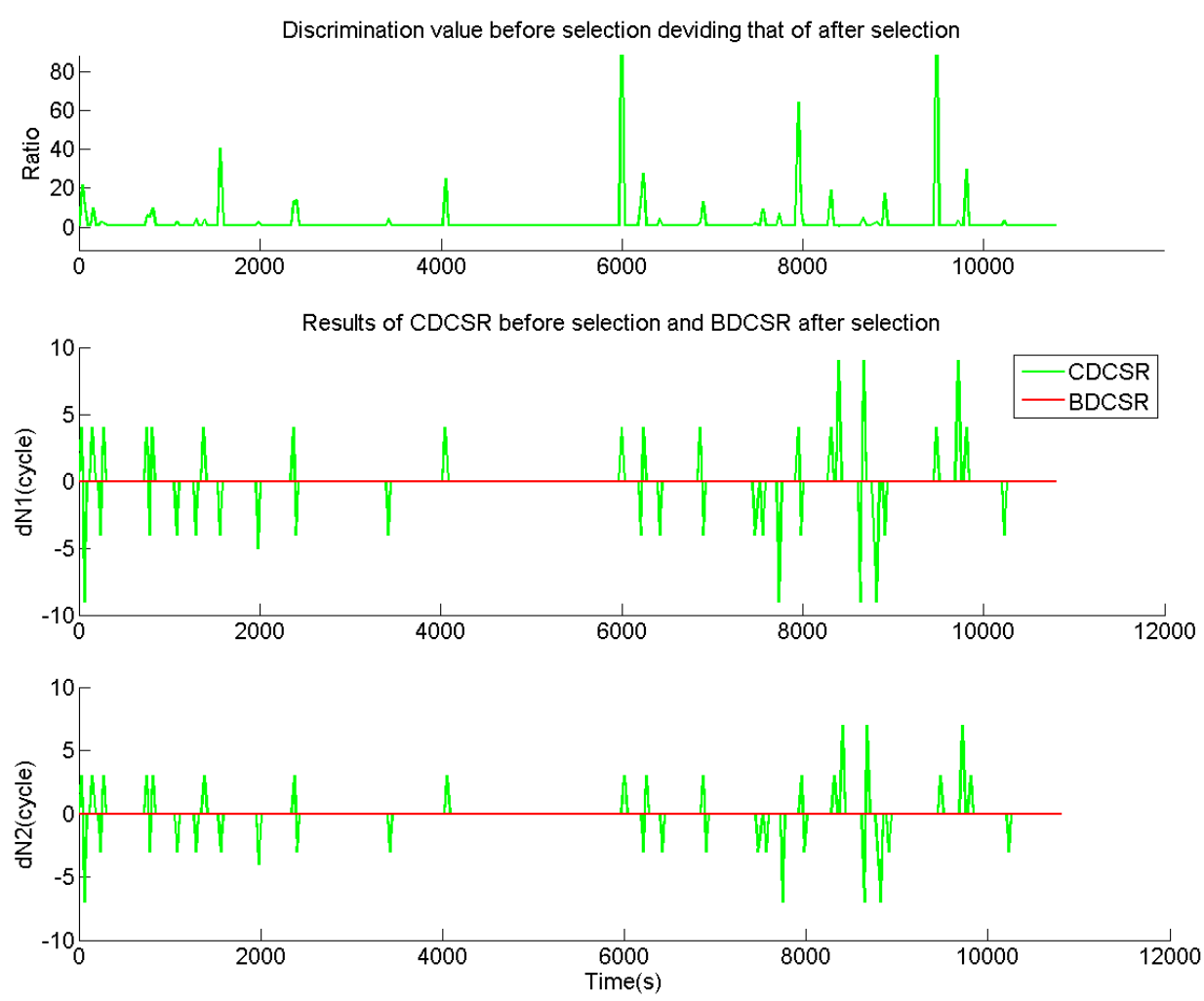

Figure 11. Results of CDCSR before selection and BDCSR after selection with the discrimination function from IGSO Satellite 6 in a weak condition of low elevation during the geomagnetic storm, where the phase data without cycle slip was collected at the CUT0 station started from UTC 8 May 2016 00:00:00 with an interval of $30 \mathrm{~s}$.

Even after $7000 \mathrm{~s}$, the elevations of MEO Satellite 11 drops from 17 degrees to 5 degrees. During this period, Figure 10 shows that many absolute values of the MW cycle-slip combinations have exceeded the tolerant limit, and many misjudgments occurred in the results of MWCSD. The absolute floats of the MW cycle-slip combinations are 1-2 cycles after 7000 s. The absolute values of the BDCSR cycle-slip combinations shown in Figure 5 are mostly within 1 cycle. Obviously, the deviated magnitude of the BDCSR cycle-slip combinations is smaller than that of the MW, so the low-level noises and high-precision cycle-slip combinations are obtained by the optimization model of the BDCSR methodology with respect to the MW.

Figure 11 shows the original cycle slips of IGSO Satellite 6 determined by the BDCSR methodology and CDCSR. It indicates that all results of the BDCSR methodology are correct. However, many results of CDCSR are wrong, and the cycle-slip values outputted from CDCSR are inconsistent with the situation of phase data without cycle slip. The reason is that large pseudorange error has significantly polluted the calculation and fixation of the cycle-slip combination, and CDCSR is unable to resist the adverse disturbance from the large pseudorange error.

4.5. CSR Results of Appending Gross Pseudorange Errors on Actual Dual-Frequency Data from MEO Satellites at Low Elevation during a Geomagnetic Storm

Experiments under the condition of real data adding gross errors on the pseudoranges were carried out so as to affirm the role of the discrimination function in eliminating the disturbance of pseudorange error. For the real data without cycle slip, a gross error of $0.7 \mathrm{~m}$ is added to all epoch-difference pseudoranges in epoch-by-epoch mode. Similarly, the real data are selected from the low-elevation MEO Satellite 11. BDCSR and CDCSR obtain the cycle-slip values and ratios shown in Figure 12. The floats of the cycle-slip combinations from the BDCSR methodology are displayed in Figure 13. MWCSD provides the floats of the MW cycle-slip combinations and CSD results exhibited in Figure 14. 

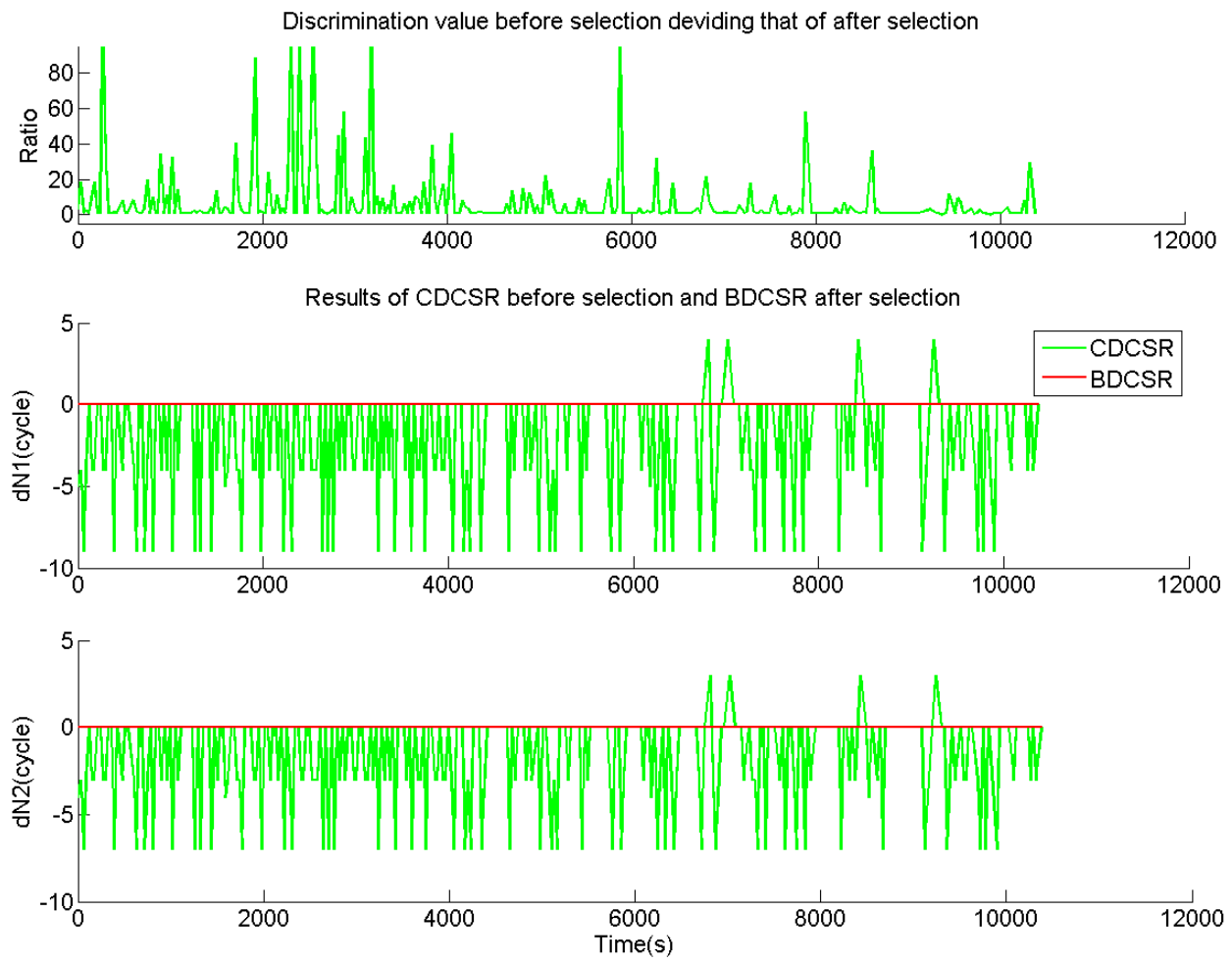

Figure 12. Results of conventional dual-frequency CSR (CDCSR) before selection and BDCSR after selection via the discrimination function in the case of appending gross errors of $0.7 \mathrm{~m}$ on all real epoch-difference pseudoranges from $\mathrm{MEO}$ Satellite 11 under a weak condition of low elevations during the geomagnetic storm, where the phase data without cycle slip was collected at the CUT0 station started from UTC 8 May 2016 00:00:00 with an interval of $30 \mathrm{~s}$.
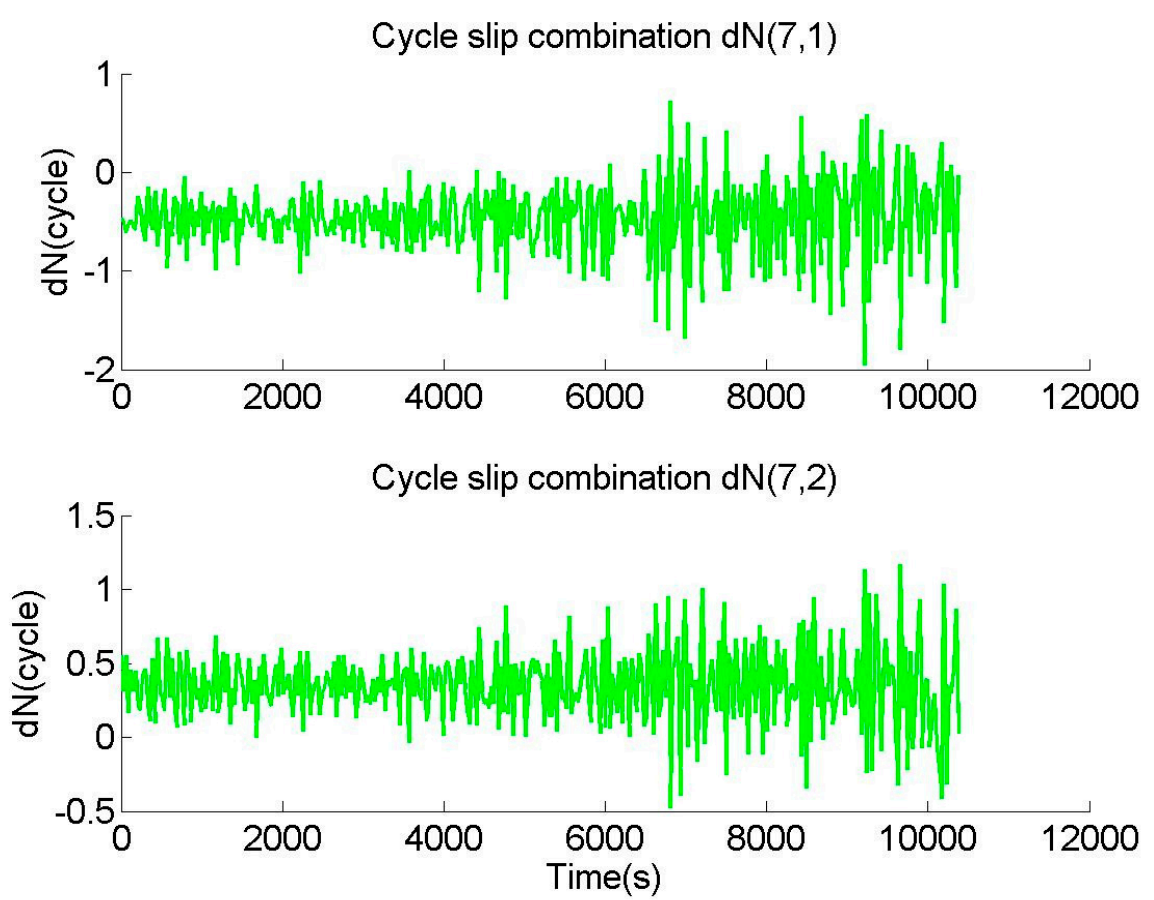

Figure 13. Floating values of the BDCSR methodology's cycle-slip combination in the case of appending gross errors of $0.7 \mathrm{~m}$ on all real epoch-difference pseudoranges from MEO Satellite 11 under a weak condition of low elevations during the geomagnetic storm, where the phase data without cycle slip was collected at the CUT0 station started from UTC 8 May 2016 00:00:00 with an interval of $30 \mathrm{~s}$. 


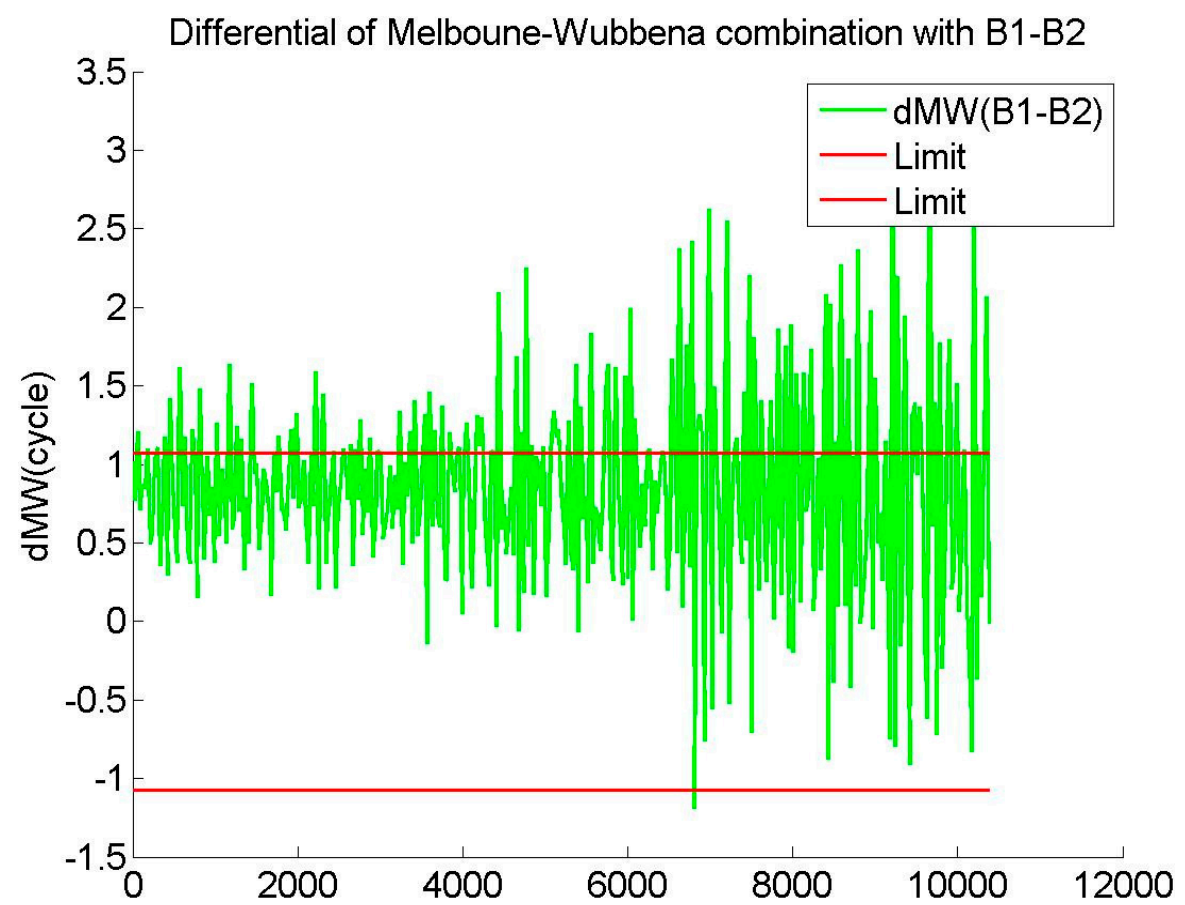

Figure 14. Floating values of the Melbourne-Wubbena combination (MW)'s cycle-slip combination in the case of appending gross errors of $0.7 \mathrm{~m}$ on all real epoch-difference pseudoranges from MEO Satellite 11 under a weak condition of low elevation during the geomagnetic storm, where the phase data without cycle slip was collected at the CUT0 station started from UTC 8 May 2016 00:00:00 with an interval of $30 \mathrm{~s}$.

Figure 12 shows the result of CDCSR before selection with the discrimination function. Results of CDCSR are almost completely wrong. Figure 13 exhibits that the floats of the BDCSR cycle-slip combination range from -2 to 1 , and most of the absolute values exceed 0.5. Hence, the directly fixed cycle-slip values from CDCSR are no longer feasible. However, the results of the BDCSR methodology after selection with the discrimination function are still correct.

Figure 14 shows the results of MWCSD processing the phase data without cycle slip, but all the real epoch-difference pseudoranges adding gross errors of $0.7 \mathrm{~m}$. It exhibits that a great number of misjudgments emerge in the results of MWCSD. Continuing to analyze the floats of the MW cycle-slip combinations, most of the values have exceeded the detection limit of MWCSD. The absolute floats of the MW cycle-slip combination deviating from the real values of 0 are between 0.5 and 3 . At this moment, the correct integer of the MW cycle-slip combination cannot be obtained from the direct fixation. Hence, the large pseudorange error has made the CSR with a single-epoch MW cycle-slip combination to be infeasible.

Summarily, the results of the BDCSR methodology remain correct in the two representative cases: the real data containing large pseudorange noise in the low-elevation environment and the aforementioned data where gross errors as large as $0.7 \mathrm{~m}$ are added on all real epoch-difference pseudoranges epoch-by-epoch. In fact, the large error makes the pseudorange to be improper to detect and fix cycle slip in a direct mode. Hence, the discrimination function is necessary and effective to exclude the harmful disturbance of pseudorange error.

\subsection{Repair Results of Small and Special Cycle Slips}

Since the small cycle slips of $(1,0)$ are hard to be handled for most CSR methods, and the special cycle slips $(1,1)$ are challenging to MWCSD. Cycle slips of $(1,0)$ and $(1,1)$ are added to all epoch-difference phases in epoch-by-epoch mode. CSR tests are carried out 
in the cases of small cycle slips and special cycle slips. BDCSR processes the phase data with the cycle slips $(1,0)$ and provides the floats of the cycle-slip combinations and the integers of the original cycle slip shown in Figure 15. In the same way, results of the BDCSR methodology processing the phase data with the cycle slips $(1,1)$ are displayed in Figure 16.
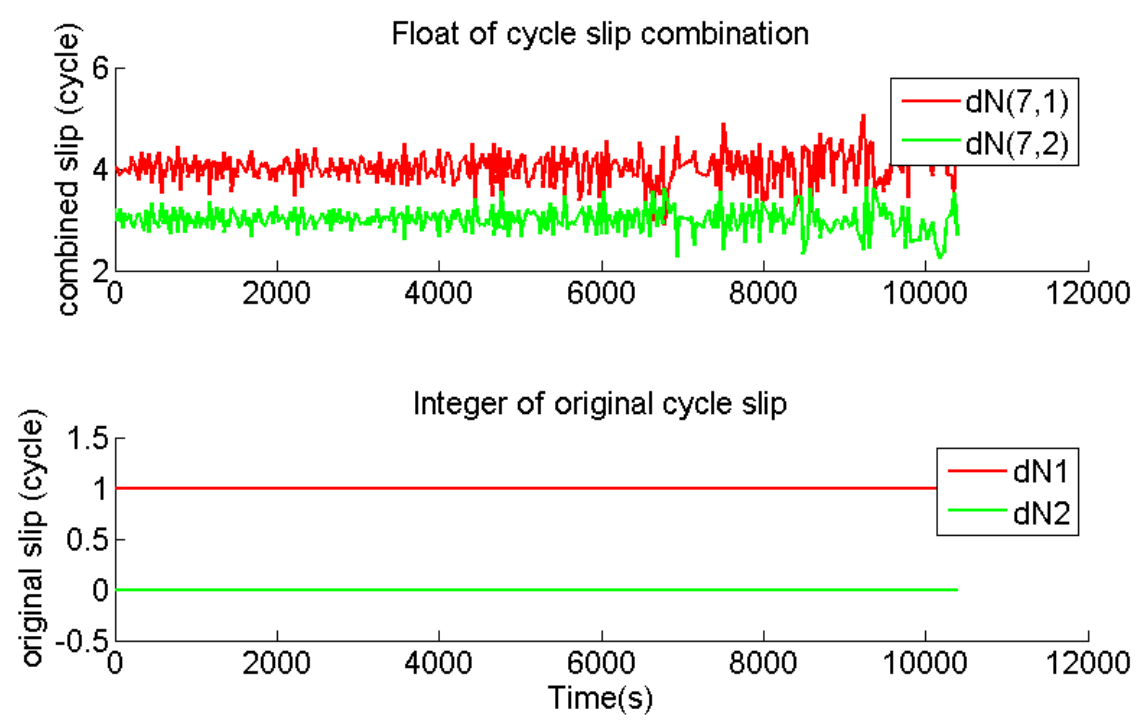

Figure 15. Results of the cycle-slip combinations and original cycle slip supplied by the BDCSR methodology from MEO Satellite 11 in a weak condition of low elevations during the geomagnetic storm, where the known cycle slips of $(1,0)$ were inserted epoch-by-epoch in the phase data collected at the CUT0 station started from UTC 8 May 2016 00:00:00 with an interval of $30 \mathrm{s.}$

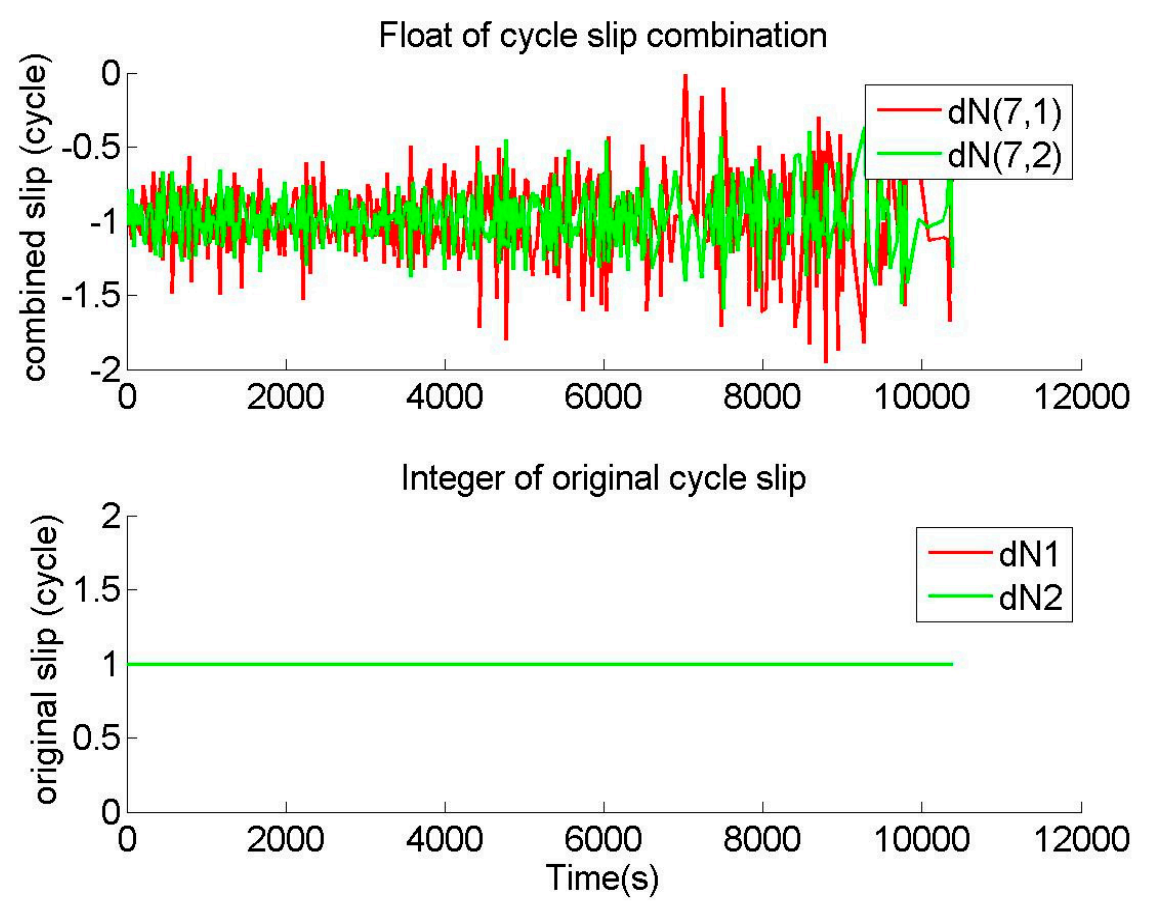

Figure 16. Results of the cycle-slip combinations and original cycle slip supplied by the BDCSR methodology from MEO Satellite 11 in a weak condition of low elevations during the geomagnetic storm, where the known cycle slips of $(1,1)$ were inserted epoch-by-epoch in the phase data collected at the CUT0 station started from UTC 8 May 2016 00:00:00 with an interval of $30 \mathrm{~s}$.

The upper parts of Figure 15 show that the floats of the BDCSR cycle-slip combinations reach up to $3-4$, and distinct responses are generated by the BDCSR methodology to the 
small cycle slips $(1,0)$. It confirms that the BDCSR methodology has a notable ability to repair small cycle slips. The bottom parts of Figure 15 show that the integers of the original cycle slip from the BDCSR methodology agree with the inserted cycle slips, and all inserted small cycle slips were correctly determined. It demonstrates that the BDCSR methodology is correct and reliable for repairing small cycle slips.

The upper parts of Figure 16 show that the response of the BDCSR cycle-slip combination to the special cycle slip of $(1,1)$ is 1 , and a valid and sensitive reaction is performed from the BDCSR methodology. However, for the cycle slips of $(\mathrm{N}, \mathrm{N})$ where $\mathrm{N}$ is any nonzero integers, the response of the MW cycle-slip combination is always 0 . Hence, MWCSD is unable to detect the cycle slips of $(\mathrm{N}, \mathrm{N})$. In contrast to the shortage of MWCSD invalid to detect special cycle slips of $(1,1)$, BDCSR can complete the CSR to the special cycle slips correctly. Nevertheless, BDCSR is unable to repair the special cycle slips of $(590,763)$ as same as the dual-frequency BDS GFCSD because the discrimination function is invalid to the cycle slips of $(590,763)$.

BDCSR and CDCSR processed the raw phase data with the cycle slips $(1,1)$ and obtained the repaired phase data. The CSR performance can be demonstrated by the epoch-difference GF values, which reflect the influence of the cycle slips on the phase data. Therefore, this test obtained the epoch-difference GFs from the raw phases with the cycle slips $(1,1)$, the repaired phases with the BDCSR or CDCSR. Figure 17 exhibits the comparisons before and after the cycle-slip repairs with the BDCSR and CDCSR. Figure 17 shows that the repaired phase data with the BDCSR methodology is free from the cycle slip. Since the epoch-difference GF from the repaired phase data with the BDCSR methodology is totally within the limit, the BDCSR methodology results are correct. However, the repaired phase data with CDCSR still presents many cycles slips in the results of GFCSD. Because several epoch-difference GFs from the repaired phase data with CDCSR exceed the limit, the CDCSR fails to repair all cycle slips correctly. Consequently, many failures occurred in CDCSR, but the performance of the BDCSR methodology is correct.

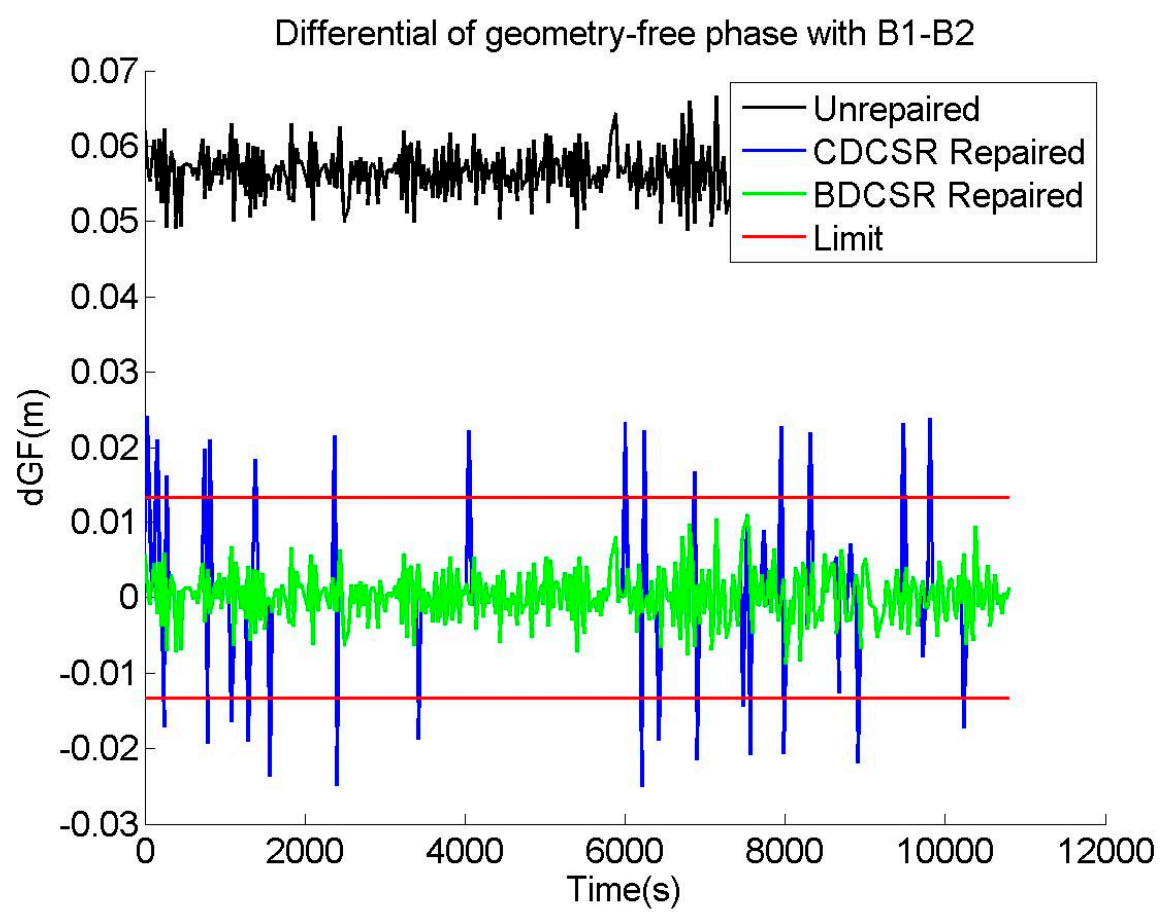

Figure 17. The differentials of geometry-free phases, provided by the unrepaired data and the repaired data with the BDCSR methodology and CDCSR in the case of normal pseudorange data and phase data with slips of $(1,1)$ cycles on $(\mathrm{B} 1, \mathrm{~B} 2)$, where the observation data were collected by the CUT0 started from UTC 8 May 2016 00:00:00. 


\subsection{Statistical Results of Different CSR Methods}

The real BDS dual-frequency data, and the real data adding gross error of $0.7 \mathrm{~m}$ on all epoch-difference pseudoranges epoch-by-epoch, were processed by GTCSR, CTCSR, WICSR, and MWCSD, respectively. The testing data were classified as 2 sets according to latitudes of stations: The one set is from medium or low latitudes of the CUT0 and ANMG, and the other set is from high latitudes of RGDG and KIRU. By counting the number of correct cycle slips and the total processed number, the actual CSR success rate is obtained. In the same way, the CSD success rate is deduced from the correctly detected number dividing the total detected number. Table 5 lists the success rates of GTCSR, CTCSR, WICSR, and MWCSD, at the medium and low latitudes of the CUT0 and ANMG during the geomagnetic storm. Furthermore, Table 6 lists the success rates of GTCSR, CTCSR, WICSR, and MWCSD, at the high latitudes of RGDG and KIRU during the geomagnetic storm.

Table 5. The success rates of various compared methods from all BDS satellites at the medium and low latitudes of the CUT0 and ANMG.

\begin{tabular}{ccc}
\hline Compared Methods & $\begin{array}{c}\text { Real Data during the } \\
\text { Geomagnetic Storm }\end{array}$ & $\begin{array}{c}\text { Real Data Adding Gross Errors of } \mathbf{0 . 7} \mathbf{~ m} \\
\text { to all Epoch-Difference Pseudoranges } \\
\text { during the Geomagnetic Storm }\end{array}$ \\
\hline MWCSD & $98.25 \%$ & $83.90 \%$ \\
WICSR & $91.57 \%$ & $53.90 \%$ \\
CDCSR & $98.15 \%$ & $56.53 \%$ \\
BDCSR (proposed) & $100.00 \%$ & $100.00 \%$ \\
\hline
\end{tabular}

Table 6. The success rates of various compared methods from all BDS satellites at high latitudes of RGDG and KIRU.

\begin{tabular}{ccc}
\hline Compared Methods & $\begin{array}{c}\text { Real Data during the } \\
\text { Geomagnetic Storm }\end{array}$ & $\begin{array}{c}\text { Real Data Adding Gross Errors of } \mathbf{0 . 7} \text { m } \\
\text { to all Epoch-Difference Pseudoranges } \\
\text { during the Geomagnetic Storm }\end{array}$ \\
\hline MWCSD & $97.86 \%$ & $80.48 \%$ \\
WICSR & $90.57 \%$ & $57.38 \%$ \\
CDCSR & $95.71 \%$ & $57.14 \%$ \\
BDCSR (proposed) & $100.00 \%$ & $100.00 \%$ \\
\hline
\end{tabular}

In the case of the real BDS dual-frequency data at medium and low latitudes during the geomagnetic storm, the statistical results in Table 5 show that the success rate of WICSR is lower than that of the BDCSR methodology. WICSR encountered an $8 \%$ failure rate because of ignoring the correction of the ionospheric variation. It indicates that the ionospheric variation should be reasonably corrected; otherwise, the calculation and fixation of the cycle-slip combination would be distorted seriously. On the other hand, CDCSR occurred $2 \%$ failure rate. The reason is that the calculation and fixation of the cycle-slip combination in the CDCSR are polluted by the large errors that occasionally occurred in the actual pseudoranges. Although the tolerant limit of MWCSD of 1.07 cycles is far larger than 0.5 cycles, there also exists a $2 \%$ failure rate in the results of MWCSD. At the same time, the results of the BDCSR methodology are correct because BDCSR has corrected the ionospheric variations and excluded the disturbance of pseudorange noise.

For the real data adding a gross error of $0.7 \mathrm{~m}$ on all epoch-difference pseudoranges epoch-by-epoch at medium and low latitudes, Table 5 exhibits that the success rate of the BDCSR methodology is higher than that of CDCSR. Since the calculation and fixation of the cycle-slip combination were gravely contaminated by large pseudorange error, there happened a $44 \%$ failure rate in the CDCSR. Similarly, many misjudgments occurred in the detection results of MWCSD, and the success rate of MWCSD was reduced to $80 \%$. However, the BDCSR methodology results remain correct. It demonstrates that the BDCSR 
methodology takes a verifiable advantage of eliminating the worse influence of large pseudorange errors so as to identify the correct cycle-slip values.

In the situation of real data from high latitudes of stations during the geomagnetic storm, the statistical results in Table 6 show that the BDCSR methodology results still correct, but WICSR encounters the failure rate of $10 \%$, CDCSR occurs the failure rate of $4 \%$, MWCSD runs into the failure rate of 3\%. Especially in the case of real data adding a gross error of $0.7 \mathrm{~m}$ on all epoch-difference pseudoranges epoch-by-epoch at high latitudes, BDCSR presents a distinct advantage to the MWCSD WICSR, CDCSR, and a significantly higher success rate is achieved by the BDCSR methodology than those of MWCSD, WICSR, CDCSR.

\section{Conclusions}

Although all BDS navigation satellites send triple-frequency signals, many BDS receivers or boards only observe and output dual-frequency phases and pseudoranges, with the consideration of low-power consumption. The phase data have been widely applied in the services, such as precise positioning, precise timing, precise orbits and clock errors determination, and so on. When the phase observation encountered an interruption, cycle slips may occur in the phase data. The cycle slip must be correctly detected and reasonably handled; otherwise, the precise positioning results will fluctuate or even fail to converge. In particular, the unrepaired cycle slips may lead to a reinitialization of the ambiguity parameter and result in additional convergence time along with precision decline.

If BDS directly applies the dual-frequency geometry-free phase (GF) and MelbourneWubbena combination (MW) widely used in GPS to conduct the cycle-slip detection (CSD) or cycle-slip repair (CSR), there will emerge many failures caused by severe ionospheric change and large pseudorange error. In response to coping with the aforementioned problems, a cycle-slip repair method for BDS dual-frequency phases (BDCSR) is developed to overcome the disturbances from severe ionospheric variation and large pseudorange error:

(1) A BDS dual-frequency optimization model is proposed to calculate cycle-slip combination, and this model reduces the influences of the observational noises and the ionospheric residuals after the correction. With the goal of minimizing the variance of the cycle-slip combination, a high-precision mode of calculating the cycle-slip combination is obtained by the BDCSR methodology with the STDs of $(0.2343,0.1710)$ cycles, which are clearly smaller than that of 0.3576 cycles in the BDS MW cycle-slip combination. Meanwhile, wavelengths of the BDCSR methodology cycle-slip combinations are $(1.4365,2.0637) \mathrm{m}$, which are much larger than that of $0.8470 \mathrm{~m}$ in BDS dual-frequency MW. On one hand, the lower STD is helpful to improve the calculated accuracy of the cycle-slip combination. On the other hand, the longer wavelength has a better suppression of the influence of pseudorange error on the calculation of the cycle-slip combination.

(2) In the BDS dual-frequency situation, a cycle-slip discrimination function is proposed to eliminate the influence of pseudorange error on the cycle-slip determination, and the actual success rate of CSR is improved. Large pseudorange error will cause tremendous bias in the calculated float of the cycle-slip combination and may result in the cycle-slip combination being fixed to the wrong integer. Synchronously, the original cycle slips transformed from the fixed cycle-slip combinations are polluted by the large pseudorange error, so CSR failures result. To overcome this problem, the absolute epoch-difference GF after correcting the ionospheric variation is designed as the discrimination function to select the final cycle slip. Since the discrimination function only retains the response to the cycle slip, the correct values of the cycle slips will lead to the minimum value of the discrimination function. By sorting the values of the discrimination function calculated with the candidates of the cycle slips, the reasonable cycle slips are selected. Hence, the discrimination function fundamentally excludes the mistakes of the cycle-slip fixation caused by the significant bias in the calculated cycle-slip combination due to the large pseudorange error. 
Many compared experiments were carried out with the real BDS dual-frequency data at the region with active ionosphere under the environment of a geomagnetic storm. The BDCSR's ability to resist the disturbances of severe ionospheric variation and large pseudorange error was verified. Experimental results demonstrate as the following: (1) CSR without correcting the ionospheric variation (WICSR) happened many mistakes, but results of the BDCSR methodology were correct due to the practical and necessary correction of ionospheric variation in the BDCSR methodology. During geomagnetic storms, cycle-slip combinations calculated with the BDS dual-frequency data from MEO and IGSO satellites have experienced a tremendous influence from the ionospheric variation. The influence of ionospheric variation is slight but intolerable in calculating the cycle-slip combination of the GEO Satellites since the relationship between the Earth, and the GEO Satellite is stationary. (2) The conventional dual-frequency CSR employing the optimized cycle-slip combinations in the absence of the discrimination function (CDCSR) occurred many mistakes under the conditions of low elevations and large pseudorange errors, while the results of the BDCSR methodology are consistent with the actual situation. (3) In further, under the condition of adding a gross error of $0.7 \mathrm{~m}$ in real epoch-difference pseudoranges, extensive failures appear in the results of CDCSR and MWCSD, but the results of the BDCSR methodology are still correct. Summarily, BDCSR achieves a higher success rate than those of WICSR, CDCSR and MWCSD. The reason is that the BDCSR methodology not only reduces the notable effect of severe ionospheric variation but also overcomes the shortcomings of MWCSD and CDCSR, that the large pseudorange errors seriously pollute the calculated values of the cycle-slip combinations and result in CSR or CSD mistakes.

Finally, BDCSR has completed the verifications under the representative conditions, such as the weather of a geomagnetic storm, the low dynamic of the GEO Satellites, the high dynamic of MEO and IGSO satellites, the fine observations at the medium elevations, the weak observations with the large pseudorange errors at low elevations, and the real observations adding gross errors to the pseudoranges. However, it is difficult for one study to fully traverse all extreme conditions and vast observation data. In the future, the work can continue to process more observation data to test the performance of the BDCSR methodology. BDCSR does not require any restrictions on the number of observable satellites, the information of navigation ephemeris, and the accessibilities of orbit and clock products. Hence, this method is simple and practical to the BDS dual-frequency data preprocessing.

Author Contributions: Conceptualization, D.L. and Y.Y.; methodology, D.L.; validation, J.M., Y.Y. and Y.D.; writing —original draft preparation, D.L.; writing—review and editing, Y.Y.; supervision, Y.D.; project administration, J.M.; funding acquisition, J.M. All authors have read and agreed to the published version of the manuscript.

Funding: We would like to gratefully acknowledge the support of the National Key Research and Development Plan of China (Grant No. 2016YFB0502105, 2016YFB0502101), the Basic scientific research of the Chinese Academy of Surveying and Mapping (Grant No. AR2006), and the National Natural Science Foundation of China (Grant No. 41304030, 41774013), for financial supports.

Institutional Review Board Statement: Not applicable.

Informed Consent Statement: Not applicable.

Data Availability Statement: The data supporting this research can be found at the hyperlinks as http:/ / saegnss2.curtin.edu.au/ldc/rinex3/daily/, accessed on 4 February 2021.

Acknowledgments: The Curtin GNSS Research Centre and the Multi-GNSS Experiment (MGEX) of the International GNSS Service (IGS) are thanked for supplying the BDS dual-frequency observation data.

Conflicts of Interest: The authors declare no conflict of interest.

\section{References}

1. Leick, A.; Rapoport, L.; Tatarnikov, D. GPS Satellite Surveying. In GPS Satellite Surveying, 4th ed.; Wiley: Hoboken, NJ, USA, 2015.

2. Xu, G. GPS Theory, Algorithms and Applications; Springer: Berlin/Heidelberg, Germany, 2007. 
3. Li, B.; Qin, Y.; Liu, T. Geometry-based cycle slip and data gap repair for multi-GNSS and multi-frequency observations. J. Geod. 2019, 93, 399-417. [CrossRef]

4. Li, T.; Melachroinos, S. An enhanced cycle slip repair algorithm for real-time multi-GNSS, multi-frequency data processing. GPS Solut. 2019, 23, 1. [CrossRef]

5. Zhang, X.; Li, X. Instantaneous re-initialization in real-time kinematic PPP with cycle slip fixing. GPS Solut. 2012, 16, $315-327$.

6. Ge, M.; Gendt, G.; Rothacher, M.; Shi, C.; Liu, J. Resolution of GPS carrier-phase ambiguities in Precise Point Positioning (PPP) with daily observations. J. Geod. 2008, 82, 389-399. [CrossRef]

7. Yang, F.; Zhao, L.; Li, L.; Cheng, J.; Zhang, J. Ionosphere-Constrained Triple-Frequency Cycle Slip Fixing Method for the Rapid Re-Initialization of PPP. Sensors 2018, 19, 117. [CrossRef] [PubMed]

8. Lacy, M.C.; Reguzzoni, M. Real-time cycle slip detection in triple-frequency GNSS. GPS Solut. 2012, 16, 353-362. [CrossRef]

9. Li, P.; Jiang, X.; Zhang, X.; Ge, M.; Schuh, H. Kalman-filter-based undifferenced cycle slip estimation in real-time precise point positioning. GPS Solut. 2019, 23, 99. [CrossRef]

10. Lacy, M.C.; Reguzzoni, M.; Sansò, F.; Venuti, G. The Bayesian detection of discontinuities in a polynomial regression and its application to the cycle-slip problem. J. Geod. 2008, 82, 527-542. [CrossRef]

11. Mertikas, S.P.; Rizos, C. On-line detection of abrupt changes in the carrier-phase measurements of GPS. J. Geod. 1997, 71, 469-482. [CrossRef]

12. Blewitt, G. An automatic editing algorithm for GPS data. Geophys. Res. Lett. 1990, 17, 199-202. [CrossRef]

13. Banville, S.; Langley, R.B. Mitigating the impact of ionospheric cycle slips in GNSS observations. J. Geod. 2013, 87, 179-193. [CrossRef]

14. Praveen, G.; Rao, S.S.; Shweta, S.; Gordiyenko, G.; Rajesh, P. Ionospheric response to the geomagnetic storm of 15 May 2005 over midlatitudes in the day and night sectors simultaneously. J. Geophys. Res. Space Phys. 2014, 119, 5020-5031.

15. Hatch, R. The Synergism of GPS Code and Carrier Measurements. In Proceedings of the Third International Symposium on Satellite Doppler Positioning at Physical Sciences Laboratory of New Mexico State University, Las Cruces, NM, USA, 8-12 February 1982; Volume 2, pp. 1213-1231.

16. Melbourne, W.G. The case for ranging in GPS-based geodetic systems. In Proceedings of the 1st International Symposium on Precise Positioning with the Global Positioning System, Rockville, ML, USA, 1 January 1985; pp. 373-386.

17. Liu, Z. A new automated cycle slip detection and repair method for a single dual-frequency GPS receiver. J. Geod. 2011, 85, 171-183. [CrossRef]

18. Cai, C.; Liu, Z.; Xia, P.; Dai, W. Cycle slip detection and repair for undifferenced GPS observations under high ionospheric activity. GPS Solut. 2013, 17, 247-260. [CrossRef]

19. Yuan, Y.; Huo, X.; Ou, J.; Zhang, K.; Chai, Y.; Wen, D.; Grenfell, R. Refining the Klobuchar ionospheric coefficients based on GPS observations. IEEE Trans. Aerosp. Electron. Syst. 2008, 44, 1498-1510. [CrossRef]

20. Li, Z.; Yuan, Y.; Fan, L.; Huo, X.; Hsu, H. Determination of the Differential Code Bias for Current BDS Satellites. IEEE Trans. Geosci. Remote Sens. 2014, 52, 3968-3979. [CrossRef]

21. Zhang, B.; Teunissen, P.J.G. Characterization of multi-GNSS between-receiver differential code biases using zero and short baselines. Sci. Bull. 2015, 60, 1840-1849. [CrossRef]

22. Wang, N.; Yuan, Y.; Li, Z.; Montenbruck, O.; Tan, B. Determination of differential code biases with multi-GNSS observations. J. Geod. 2016, 90, 209-228. [CrossRef]

23. Teunissen, P.J.G. The least-square ambiguity decorrelation adjustment: A method for fast GPS ambiguity estimation. J. Geod. 1995, 70, 65-82. [CrossRef] 